H. Beirão da Veiga

\title{
On the Ladyzhenskaya-Smagorinsky turbulence model of the Navier-Stokes equations in smooth domains. The regularity problem
}

Received October 10, 2006 and in revised form June 29, 2007

\begin{abstract}
We establish regularity results up to the boundary for solutions to generalized Stokes and Navier-Stokes systems of equations in the stationary and evolutive cases. Generalized here means the presence of a shear dependent viscosity. We treat the case $p \geq 2$. Actually, we are interested in proving regularity results in $L^{q}(\Omega)$ spaces for all the second order derivatives of the velocity and all the first order derivatives of the pressure. The main aim of the present paper is to extend our previous scheme, introduced in [3] and [4] for the flat-boundary case, to the case of curvilinear boundaries.
\end{abstract}

\section{Introduction}

In the sixties Olga Ladyzhenskaya proposed a new set of equations conceived in order to build a model for turbulence phenomena. A typical model is the following:

$$
\left\{\begin{array}{l}
\partial_{t} u+(u \cdot \nabla) u-\nabla \cdot \mathbb{T}(u, \pi)=f, \\
\nabla \cdot u=0,
\end{array}\right.
$$

in $\Omega \times] 0, T$, where $\mathbb{T}$ denotes the stress tensor

$$
\begin{gathered}
\mathbb{T}=-\pi I+v_{T}(u) \mathcal{D} u, \quad \mathcal{D} u=\frac{1}{2}\left(\nabla u+\nabla u^{T}\right), \\
v_{\mathbb{T}}(u)=v_{0}+v_{1}|\mathcal{D} u|^{p-2},
\end{gathered}
$$

and $v_{0}, v_{1}$ are strictly positive constants. This system satisfies the Stokes principle, a fundamental physical requirement (see Appendix 1).

This new model prescribes that the stress tensor $\mathbb{T}$ depends on the symmetric part $\mathcal{D} u$ of the gradient of the velocity in a nonlinear, polynomial way, with $p$-rate of growth, for $p>2$ (as originally proposed). By setting $p=3$, we get the classical Smagorinsky model, introduced by him in [32], as a turbulence model (see also [13] and [27]). In [20] and [21, Chap. 2, n. 5], J.-L. Lions considers the case in which $\mathcal{D} u$ is replaced by $\nabla u$. However, in this case the Stokes principle is not satisfied.

H. Beirão da Veiga: Dipartimento di Matematica Applicata “U. Dini”, Via Buonarrotti, 1/C, I-56127 Pisa, Italy; e-mail: bveiga@dma.unipi.it

Mathematics Subject Classification (2000): 35J25, 35Q30, 76D03, 76D05 
One of the features of the above $p$-rate of growth was to prove existence and uniqueness of solutions, taking advantage of such an increased coercivity; indeed the nonlinearity induced by the $(u \cdot \nabla) u$ term is not any longer critical when $p$ is assumed to be large enough. This overcomes the lack of the corresponding solvability property of the Navier-Stokes equations. Such kind of models were intensively investigated in the eighties and nineties by J. Nečas and his school, in order to study certain particular kinds of fluids, and in particular to describe shear thickening $(p>2)$ and shear thinning $(p<2)$ phenomena. In particular, $L^{q}$ regularity results, up to the boundary, for the second order derivatives of the solutions $u$ to Ladyzhenskaya type models in bounded domains $\Omega$, for $p>2$ and under the nonslip boundary condition

$$
u_{\mid \Gamma}=0
$$

are stated in [23].

Peculiar features of certain fluids can indeed be described in a better way using polynomial, rather than linear, growth conditions on the stress tensor. We refer the reader to the basic book [22], particularly relevant for fluids of the type analyzed in this paper. We also refer to [12] and [28].

The mathematical analysis concerning the above kind of models is far from being trivial, and usually involves a large amount of delicate arguments of both technical and substantial nature. We are mainly interested in the question of integrability up to the boundary, with some exponent, of the second derivatives of the velocity and the first derivatives of the pressure. In [3] we give a solution to this problem for half-space, in the stationary case. See also [4]. The scheme proposed in [3] is as follows. At first, the steady state problem with no convection term is considered. It is a kind of nonlinear Stokes problem. For $p \in[2,3]$, characterizing the growth of the dissipative potential, the starting regularity is proved. More precisely, it is shown that the second derivatives of the velocity field are integrable with power $p^{\prime}=p /(p-1)$. Then this exponent is improved up to some exponent $l>p^{\prime}$, if $p<3$, by bootstrap arguments. For $p=2$, the results are the same as in the case of the classical Stokes problem with the right hand side in $L^{2}$. Further, all the results remain true in the presence of a convective term. For $p>2$, its role is not so crucial.

The aim of the present paper is to extend the above scheme to the case of curvilinear boundaries. This is a quite difficult technical problem, especially for nonlinear equations containing a viscosity term depending on the modulus of the symmetric part of the gradient. Actually, the latter circumstance makes the problem difficult even in the case of flat boundaries. The known scheme developed for the case in which the coefficients in the equations depend on the modulus of the gradient (Lions model) does not work here (see Remark 3.1 below). The proof is done via a careful analysis up to the boundary, and a suitable application of a modified difference quotient method (which is the novelty of the paper) overcoming the simultaneous appearance of three difficulties: boundary regularity (that is, how to recover the vertical derivatives of $\mathcal{D} u$ from the tangential ones), the divergence constraint to be met at each choice of the test functions, and the fact that the system actually depends on the symmetric part of the gradient, rather than on the gradient itself. This leads to the introduction of a certain number of interesting new tricks. The results 
are anyway proved by first arguing locally, via a suitable flattening of the boundary, and then by a covering argument to recover the final global estimate. The main scientific importance of our work could be that all the results that are valid for flat boundaries remain valid for general smooth domains.

Nowadays, the electrorheological fluids are a class of fluids of interest. We refer the reader to [30], and also to [29] where the original modeling developed in [30] is proposed. See also [1] for a detailed analysis for nonsimplified models in the stationary case. A similar modeling, using a variable exponent structure, is also proposed in [34]. Other interesting models are proposed, for instance, in [24] and [8]-[10].

\section{Main results}

In the following, $\Omega$ is a bounded, connected, open set in $\mathbb{R}^{3}$, locally situated on one side of its boundary

$$
\Gamma=\partial \Omega
$$

a manifold of class $C^{2}$. Further, we assume that $p \geq 2$.

We consider generalized Stokes and Navier-Stokes equations in the stationary case and in the evolution case, where the viscosity is given by $(1.3)$. The very basic results are those proved for the generalized Stokes stationary problem

$$
\left\{\begin{array}{l}
-\nabla \cdot\left(v_{0} \mathcal{D} u+v_{1}|\mathcal{D} u|^{p-2} \mathcal{D} u\right)+\nabla \pi=f, \\
\nabla \cdot u=0
\end{array}\right.
$$

Note that $\nabla \cdot(\mathcal{D} u)=\frac{1}{2} \Delta u$.

Before stating our main results let us introduce some simplified notation. Weak solutions satisfy the estimates 4.3 and 4.5 below. Hence, given $f \in L^{2}(\Omega)$, the quantities $\|\nabla u\|_{p}$ and $\|\pi\|_{p^{\prime}}$ are bounded. For convenience we denote by $P$,

$$
P=P\left(\|\nabla u\|_{p},\|\pi\|_{p^{\prime}}\right),
$$

very simple expressions that depend only on the two quantities indicated above. In particular, these quantities are independent of the exponent $q$ that appears in the inclusion 2.4. Explicit expressions for these quantities follow immediately from our calculations (we will write explicit expressions up to a certain point).

Concerning the lack of dependence on the exponent $q$, it is worth noting that in the following $2 \leq p \leq 3$ and $p \leq q \leq 6$ (more precisely, for a fixed $p$ the exponent $q$ lies in the range $p \leq q \leq q_{\infty}=3(4-p)$ ). In particular, Sobolev embedding constants and similar (used in the following) are bounded by constants which are independent of $q$ in the above ranges.

\subsection{The stationary problem}

Concerning the stationary case, we prove three main theorems. Theorem 2.1, which can be considered as a "starting point", ensures the global higher differentiability of the solution. 
Theorem 2.1. Let $2 \leq p \leq 3$. Assume that

$$
f \in L^{2}(\Omega)
$$

and let $(u, \pi)$ be the weak solution to problem (2.1) under the boundary condition (1.4). Assume, in addition, that

$$
\mathcal{D} u \in L^{q}(\Omega)
$$

for some $p \leq q \leq 6$. Then

$$
\|u\|_{W^{2, r}(\Omega)}+\|\pi\|_{L^{r}(\Omega)} \leq C\left(1+\|\nabla u\|_{q}^{(p-2) / 2}\right)\left(P+\|f\|_{2}\right)
$$

and

$$
\|\nabla \pi\|_{L^{\bar{p}}(\Omega)} \leq C\left(1+\|\nabla u\|_{q}^{p-2}\right)\left(P+\|f\|_{2}\right)
$$

where $r=r(p, q)$ and $\bar{p}=\bar{p}(p, q)$ are given by

$$
\frac{1}{r}=\frac{p-2}{2 q}+\frac{1}{2}, \quad \frac{1}{\bar{p}}=\frac{p-2}{q}+\frac{1}{2} .
$$

Note that at this stage the assumption $\mathcal{D} u \in L^{q}$ is only satisfied with $q=p$, since a solution, by standard monotonicity methods, can be initially found in $W^{1, p}$. Therefore the application of this result with $q=p$ leads to the following intermediate regularity result.

Theorem 2.2. Let $2 \leq p \leq 3$. Assume that

$$
f \in L^{2}(\Omega)
$$

and let $(u, \pi)$ be the weak solution to problem (2.1) under the boundary condition (1.4). Then

$$
\|u\|_{W^{2, p^{\prime}}(\Omega)}+\|\pi\|_{L^{p^{\prime}}(\Omega)} \leq C\left(1+\|\nabla u\|_{p}^{(p-2) / 2}\right)\left(P+\|f\|_{2}\right)
$$

and

$$
\|\nabla \pi\|_{L^{p_{0}(\Omega)}} \leq C\left(1+\|\nabla u\|_{p}^{p-2}\right)\left(P+\|f\|_{2}\right),
$$

where $p_{0}=2 p / 3 p-4$. Note that both the above right hand sides are bounded by an expression of the form $P(1+\|f\|)$.

The next step, which leads to the main regularity result of the paper, is to iterate Theorem 2.1 starting from Theorem 2.2 as follows: Theorem 2.2 allows one to get higher integrability of $\mathcal{D} u$ via standard Sobolev embedding, say $\mathcal{D} u \in L^{q}$; then Theorem 2.1 yields higher integrability of the second derivatives; this in turn implies higher integrability of $\mathcal{D} u$ and so on. Note that this kind of iteration usually leads to establishing higher integrability for every exponent strictly less than a certain limiting one, say $l$. Actually, the exponent $l$ can be reached since in Theorems 2.1 and 2.2 explicit boundary estimates are provided, in turn allowing for a precise control of the constants in the iteration procedure. Getting such precise estimates requires considerable technical efforts. We have the following result. 
Theorem 2.3. Let $2 \leq p \leq 3$, and let $f, u$ and $\pi$ be as in Theorem 2.2 Then

$$
\|u\|_{W^{2, l}(\Omega)} \leq C\left(P+\|f\|_{2}\right)+\left(P+\|f\|_{2}\right)^{2 /(4-p)} \leq P\left(1+\|f\|_{2}^{2 /(4-p)}\right),
$$

where

$$
l=l(p)=3 \frac{4-p}{5-p}
$$

and

$$
\|\nabla \pi\|_{m} \leq P\left(1+\|f\|^{p /(4-p)}\right),
$$

where

$$
m=m(p)=\frac{6(4-p)}{8-p} .
$$

The above iteration process requires that $p<3$. For $p=3$, the proof of Theorem 2.3 is much simpler, by following the proof of Theorem 2.1 with $q=p$. We point out that the main lines of our proofs apply as well to $p<4$, with some quite obvious modifications.

Note that the exponent $l$ turns out to be just the exponent for which Theorem 2.1, with $q=l^{*}$ (see (8.3) $)$, yields $u \in W^{2, l}$. Then, by the Sobolev embedding theorem, $u \in W^{1, l^{*}}$. In other words, $q=l^{*}$ is the fixed point of the map $q \mapsto r \mapsto r^{*}$. So, further regularity cannot be obtained by appealing to Theorem 2.1 .

When $p=2$, the statements and estimates established in Theorems 2.2 and 2.3 coincide with the classical results for the linear Stokes problem.

As shown in the proofs below, the above regularity theorems hold locally. More precisely, if $(u, \pi)$ is a weak solution in some neighborhood of a point $x_{0} \in \Gamma$ then the regularity results hold, say, in a neighborhood of one-half the radius.

The extension to the stationary generalized Navier-Stokes system 2.15 below is straightforward. We prove the following result.

Theorem 2.4. All the regularity results stated in Theorems 2.12 .3 hold for the generalized Navier-Stokes equations

$$
\left\{\begin{array}{l}
-\frac{\nu_{0}}{2} \Delta u-v_{1} \nabla \cdot\left(|\mathcal{D} u|^{p-2} \mathcal{D} u\right)+u \cdot \nabla u+\nabla \pi=f, \\
\nabla \cdot u=0 .
\end{array}\right.
$$

Moreover, the estimates in the above statements hold provided that $\|f\|$ is replaced by $P\|f\|$.

\subsection{The evolution problem}

Finally, we turn to the evolutionary case:

$$
\left\{\begin{array}{l}
\partial_{t} u+(u \cdot \nabla) u-v_{0} \nabla \cdot \mathcal{D} u-v_{1} \nabla \cdot\left(|\mathcal{D} u|^{p-2} \mathcal{D} u\right)+\nabla \pi=f \\
\nabla \cdot u=0 \\
u(0)=u_{0}(x)
\end{array}\right.
$$


The regularity up to the boundary of the second order derivatives in the time-dependent case is easily reduced to a steady state problem in which the solution depends on $t$ as a parameter, and the derivative in $t$ is considered as part of the right hand side. This is why (in the presence of the convective term) an additional restriction on $p$ comes up: $p \leq 2+2 / 5$. This restriction provides solutions $u$ such that $\partial_{t} u \in L^{2}\left(0, T ; L^{2}(\Omega)\right)$, a well known result, the proof of which is straightforward. See, for instance, Theorem 7.2 in [18]. Note that $L^{2}(\Omega)$ is exactly the regularity assumed here for the external force field $f$. This situation allows us to consider $\partial_{t} u$ as part of the right hand side of our main equation, and hence allows us to regularize the solution with respect to the space variables simply by appealing to our regularity theorems for stationary solutions.

We prove the following results.

Theorem 2.5. Let $u$ be a weak solution to problem 2.16) under the boundary condition (1.4), where $u_{0} \in V_{p}$ and $f \in L^{2}\left(0, T ; L^{2}\right)$. Assume that

$$
2+\frac{2}{5} \leq p \leq 3
$$

Then

$$
\left\{\begin{array}{l}
u \in L^{2}\left(0, T ; W^{2, p^{\prime}}\right) \cap L^{\infty}\left(0, T ; W^{1, p}\right), \\
\nabla \pi \in L^{2}\left(0, T ; L^{p_{0}}\right), \\
\partial_{t} u \in L^{2}\left(0, T ; L^{2}\right) .
\end{array}\right.
$$

The first assumption in (2.17) is not necessary if the convective term is not present in the equations.

Theorem 2.6. Under the assumptions of Theorem 2.5 one has

$$
\left\{\begin{array}{l}
u \in L^{4-p}\left(0, T ; W^{2, l}\right) \cap L^{\infty}\left(0, T ; W^{1, p}\right), \\
\nabla \pi \in L^{2(4-p) / p}\left(0, T ; L^{m}\right), \\
\partial_{t} u \in L^{2}\left(0, T ; L^{2}\right) .
\end{array}\right.
$$

The assumption 2.17) is not necessary if the convective term is not present in the equations.

\section{Remarks}

Remark 3.1 (On the regularity up to the boundary). In going from interior to boundary regularity, when $p \neq 2$, quite specific obstacles appear, unusual in other typical problems of mathematical fluid mechanics. An indication of this situation (see [3] and [4]) is the lower regularity obtained for the normal derivatives in comparison to that obtained for the tangential derivatives. Note the following. In proving interior regularity by the classical translation method (see [26]), the translations are admissible in all the $n$ independent directions. This allows suitable $L^{2}$ estimates for $\nabla \mathcal{D} u$, where the full gradient $\nabla$ is obtained thanks to the possibility of appealing to translations in all directions. On the other hand, 
it is easily shown that $c|\nabla \nabla u| \leq|\nabla \mathcal{D} u| \leq C|\nabla \nabla u|$. These two facts together lead to a small distinction if we replace $\mathcal{D} u$ by $\nabla u$ in the expression of the stress tensor. However, in proving regularity up to the boundary, the two cases are completely distinct. In fact, solutions to the J.-L. Lions model (where $\mathcal{D} u$ is replaced by $\nabla u$ ) belong to $W^{2,2}$ up to the boundary.

Summarizing, it seems not accidental that there is a very extensive literature on interior regularity for the above class of problems but, as far as we know, scant literature concerning regularity up to the boundary, in the 3-D case.

Remark 3.2 (The slip boundary condition). In [3] we prove regularity results also for solutions to the slip (or Navier) boundary condition. The same extension can be done here in the case of an arbitrary regular boundary $\Gamma$. This requires just a more careful transformation formula for vector fields under local changes of coordinates (5.2). In fact, in the following, we change each of the three components of a vector field as if they were scalars. This is sufficient under the boundary condition (1.4) since the constraint $u=0$ on the boundary is preserved by the above transformation. However, in the case of slip boundary conditions, the constraint that should be preserved is the tangency to the boundary. Hence, in order to extend our results to the slip boundary condition we should use the covariant transform of a vector field $v$ given by

$$
\tilde{v}_{j}(y)=v_{j}, \quad \widetilde{v}_{3}(y)=v_{3}-\left(\partial_{1} h\right) v_{1}-\left(\partial_{2} h\right) v_{2}
$$

where $j=1,2$, and the $v_{i}$ functions are calculated at the point $\left(y^{\prime}, y_{3}+h\left(y^{\prime}\right)\right)$ (the notation is that introduced below). See equation (4.5) in [3]. We believe that in this case there is not a substantial difference in the calculations to be done. However, they become more elaborate.

For the use of the transformation (3.1) under the slip boundary condition, we also refer the reader to [2].

Remark 3.3 (The case $v_{0}=0$ ). In the proof presented below, the presence of the term $-\Delta u$ is necessary. However, by appealing to a new device (see Remark 5.1 in [4]; see also [5], where the case $p<2$ is considered), we may prove all the results stated in Section 2 even if $v_{0}=0$, provided that the term $|\mathcal{D} u|^{p-2}$ is replaced (for instance) by $(1+|\mathcal{D} u|)^{p-2}$.

Remark 3.4 (Further improvements). Concerning the flat boundary case, very recently (after this paper was accepted by the editors), the results stated in [4] have been improved in two forthcoming papers, namely, [11] and [6]. In this last reference we show that $u \in W^{2, l} \cap W^{1, p+4}$, where $l=3(p+4) /(p+1)$. Further, the method introduced in the present paper allows the extension of these improvements from flat boundaries to curvilinear boundaries. In other words, Theorems 2.4 and 2.6 still hold with the above better exponents. 


\section{Weak solutions}

Notation. The symbol $\|\cdot\|_{p}$ denotes the canonical norm in $L^{p}(\Omega)$. Further, $\|\cdot\|=\|\cdot\|_{2}$. We denote by $W^{k, p}(\Omega), k$ a positive integer and $1<p<\infty$, the usual Sobolev space of order $k$, by $W_{0}^{1, p}(\Omega)$ the closure in $W^{1, p}(\Omega)$ of $C_{0}^{\infty}(\Omega)$, and by $W^{-1, p^{\prime}}(\Omega)$ the strong dual of $W_{0}^{1, p}(\Omega)$, where $p^{\prime}=p /(p-1)$. The canonical norms in these spaces are denoted by $\|\cdot\|_{k, p}$. Moreover, $L_{\#}^{p}(\Omega)$ denotes the subspace of $L^{p}$ consisting of the functions with vanishing mean value.

In notation concerning duality pairings and norms, we will not distinguish between scalar and vector fields. Very often we also omit from the notation the symbols indicating the domains $\Omega$ or $\Gamma$, provided that the meaning remains clear.

We set

$$
\mathbb{L}^{p}\left(\Omega_{0}\right)=\left[L^{p}\left(\Omega_{0}\right)\right]^{3}, \quad \mathbb{W}^{k, p}\left(\Omega_{0}\right)=\left[W^{k, p}\left(\Omega_{0}\right)\right]^{3}, \quad \mathbb{W}_{0}^{1, p}\left(\Omega_{0}\right)=\left[W_{0}^{1, p}\left(\Omega_{0}\right)\right]^{3},
$$

for any open subset $\Omega_{0}$ of $\mathbb{R}^{3}$. We remark that $\|\mathcal{D} v\|_{p}$ is a norm in $\mathbb{W}_{0}^{1, p}$, which is equivalent to the canonical norm.

We set

$$
V_{p}=\left\{v \in W^{1, p}(\Omega):(\nabla \cdot v)_{\mid \Omega}=0 ; v_{\mid \Gamma}=0\right\} .
$$

Note that, by appealing to inequalities of Korn's type, one shows that there is a positive constant $c$ such that

$$
\|\nabla v\|_{p}+\|v\|_{p} \leq c\|\mathcal{D} v\|_{p}
$$

for each $v \in V_{p}$. Hence the two quantities above are equivalent norms in $V_{p}$.

We denote by $c, \bar{c}, c_{1}, c_{2}$, etc., positive constants that depend, at most, on $\Omega, v_{0}, v_{1}$ and $p$. Nevertheless we allow the constants $v_{0}$ and $v_{1}$ to appear each time this helps to understand the passage from one equation to the next one.

The dependence of the constants $c$ on $p$ is not crucial provided that $1<p_{0} \leq p \leq$ $p_{1}<\infty$.

The same symbol $c$ may denote different constants, even in the same equation.

Definition. We say that a pair $(u, \pi)$ is a weak solution of problem [2.1), (1.4) if it belongs to $\mathbb{W}_{0}^{1, p}(\Omega) \times L_{\#}^{p^{\prime}}(\Omega)$ and satisfies

$$
\begin{aligned}
\frac{\nu_{0}}{2} \int_{\Omega} \nabla u \cdot \nabla \phi d x+ & v_{1} \int_{\Omega}|\mathcal{D} u|^{p-2} \mathcal{D} u \cdot \mathcal{D} \phi d x \\
& -\int_{\Omega} \pi(\nabla \cdot \phi) d y+\int_{\Omega}(\nabla \cdot u) \psi d x=\int_{\Omega} f \cdot \phi d x .
\end{aligned}
$$

for $\operatorname{each}(\phi, \psi) \in \mathbb{W}_{0}^{1, p}(\Omega) \times L_{\#}^{p^{\prime}}(\Omega)$.

Since a solution $u$ of 4.2 necessarily satisfies

$$
\int_{\Omega} \nabla \cdot u d x=0,
$$

it readily follows that 4.2 holds for each $(\phi, \psi) \in \mathbb{W}_{0}^{1, p}(\Omega) \times L^{p^{\prime}}(\Omega)$. 
Existence and uniqueness of the above solution is well known; see [22]. By setting $(\phi, \psi)=(u, \pi)$ in the above definition we show that weak solutions satisfy, in particular, the estimates

$$
\left\{\begin{array}{l}
v_{0}\|\nabla u\| \leq c\|f\|, \\
v_{1}\|\nabla u\|_{p} \leq c\|f\|_{p^{\prime}}^{1 /(p-1)} .
\end{array}\right.
$$

Moreover, by setting in $4.2 \psi=0$ and by using test functions $\phi \in C_{0}^{\infty}(\Omega)$ one gets

$$
\nabla \pi=-\nabla \cdot\left[v_{0} \nabla u+v_{1}|\mathcal{D} u|^{p-2} \mathcal{D} u\right]+f .
$$

By appealing to a classical result of Nečas we prove that

$$
\|\pi\|_{L^{p^{\prime}}} \leq c\left(v_{0}\|\nabla u\|+v_{1}\|\mathcal{D} u\|_{p}^{p-1}+\|f\|_{p^{\prime}}\right) .
$$

For convenience we fix $\pi$ by assuming that its mean value in $\Omega$ vanishes.

Now let $\Omega_{0} \subseteq \Omega$ be an arbitrary open subset of $\Omega$ (in particular $\Omega$ itself), assume that $\nabla u \in L^{s}(\Omega)$, set $\psi=0$ and use test functions $\phi \in C_{0}^{\infty}\left(\Omega_{0}\right)$. Then

$$
\|\pi\|_{L_{\#}^{s /(p-1)}} \leq c\left(v_{0}\|\nabla u\|_{s /(p-1)}+v_{1}\|\mathcal{D} u\|_{S}^{p-1}+\|f\|_{2}\right),
$$

where the norms relate to $\Omega_{0}$ and $s /(p-1) \leq 6$. In particular,

$$
\|\pi\|_{L^{s /(p-1)}} \leq c\left(v_{0}\|\nabla u\|_{s /(p-1)}+v_{1}\|\mathcal{D} u\|_{s}^{p-1}+\|f\|_{2}+\|\pi\|_{p^{\prime}}\right)
$$

in any $\Omega_{0} \subseteq \Omega$.

\section{The change of variables}

In order to reduce our problem, by a suitable change of variables, to a problem involving a flat boundary, we need to consider functions with a sufficiently small support.

Let $x_{0} \in \Gamma$ be given and let $\Pi$ be the tangent plane to $\Gamma$ at $x_{0}$. We assume that the $x_{i}$ axes, $i=1,2,3$, are such that the origin coincides with $x_{0}$, and the $x_{3}$ axis has the direction of the inward normal to $\Gamma$ at $x_{0}$. Hence the $x_{i}$ axes, $i=1,2$, lie in the plane $\Pi$. We may use this particular system of coordinates since the analytical expressions that appear on the left hand side of (4.2) are invariant under orthogonal transformations, due to the invariance of the analytical expressions of the divergence and gradient.

We assume that $\Gamma$ is a manifold of class $C^{2}$. Let $x_{0} \in \Gamma$ be given and let $\left(x^{\prime}, x_{3}\right)=$ $\left(x_{1}, x_{2}, x_{3}\right)$ be the above system of coordinates. There is a positive real $a$ and a real function $x_{3}=\eta\left(x^{\prime}\right)$ of class $C^{2}$ defined on the sphere $\left\{x^{\prime}:\left|x^{\prime}\right|<a\right\}$ such that: the points $x$ for which $x_{3}=\eta\left(x^{\prime}\right)$ belong to $\Gamma$; the points $x$ for which $\eta\left(x^{\prime}\right)<x_{3}<a+\eta\left(x^{\prime}\right)$ belong to $\Omega$; and the points $x$ for which $-a+\eta\left(x^{\prime}\right)<x_{3}<\eta\left(x^{\prime}\right)$ belong to $\mathbb{R}^{3}-\Omega$. Without loss of generality, we assume that $a \leq 1$. We define

$$
\begin{aligned}
I_{a} & =\left\{x:\left|x^{\prime}\right|<a,-a+\eta\left(x^{\prime}\right)<x_{3}<a+\eta\left(x^{\prime}\right)\right\}, \\
\Omega_{a} & =\left\{x \in I_{a}: \eta\left(x^{\prime}\right)<x_{3}\right\}, \quad \Gamma_{a}=\left\{x \in I_{a}: x_{3}=\eta\left(x^{\prime}\right)\right\} .
\end{aligned}
$$

Clearly $\Omega_{a}=\Omega \cap I_{a}$ and $\Gamma_{a}=\Gamma \cap I_{a}$. 
Actually, we extend the function $\eta\left(x^{\prime}\right)$ to the whole of $\Omega_{a}$ by setting $\eta\left(x^{\prime}, x_{3}\right)=$ $\eta\left(x^{\prime}\right)$. Nevertheless, since $\eta$ is independent of $x_{3}$, we use the notation $\eta\left(x^{\prime}\right)$.

Next we introduce the change of variables $y=T x$ given by

$$
\left(y_{1}, y_{2}, y_{3}\right)=\left(x_{1}, x_{2}, x_{3}-\eta\left(x^{\prime}\right)\right), \quad\left(x_{1}, x_{2}, x_{3}\right)=\left(y_{1}, y_{2}, y_{3}+\eta\left(y^{\prime}\right)\right),
$$

and set

$$
\begin{aligned}
J_{a} & =\left\{y:\left|y^{\prime}\right|<a,-a<y_{3}<a\right\}, \\
Q_{a} & =\left\{y \in J_{a}: 0<y_{3}\right\}, \quad \Lambda_{a}=\left\{y \in J_{a}: y_{3}=0\right\} .
\end{aligned}
$$

The map $T$ is a $C^{2}$ diffeomorphism of $I_{a}$ onto $J_{a}$ that maps $\Omega_{a}$ onto $Q_{a}$ and $\Gamma_{a}$ onto $\Lambda_{a}$. Note that the Jacobian determinant of the map $T$ is equal to 1 .

We define functions $\widetilde{g}$ by setting $\widetilde{g}(y)=g(x)$ or, more precisely, by

$$
\widetilde{g}(y)=g\left(T^{-1}(y)\right),
$$

where $g$ denotes an arbitrary scalar or vector field. As a notational rule, $g=g(x)$ and $\widetilde{g}=$ $\widetilde{g}(y)$. Moreover, partial derivatives and differential operators when applied to functions $g$ concern the $x$ variables and when applied to functions $\widetilde{g}$ concern the $y$ variables. We write $\partial_{k} g$ instead of $\partial g / \partial x_{k}$. Hence

$$
\partial_{k} \tilde{g}=\frac{\partial \widetilde{g}(y)}{\partial y_{k}} \quad \text { and } \quad \partial_{k} g=\frac{\partial g(x)}{\partial x_{k}} .
$$

Note the distinction between $\widetilde{\nabla f}$ and $\nabla \widetilde{f}$. Actually, $\widetilde{\nabla f}(y)=\left(\nabla_{x} f\right)\left(T^{-1}(y)\right)$ and $(\nabla \widetilde{f})(y)=\nabla_{y}\left[f\left(T^{-1}(y)\right)\right]$.

Since some expressions are quite long, in addition to the "tilde" notation we also use the symbol $\mathcal{T}$ for the map $f \mapsto \widetilde{f}$. In other words,

$$
(\mathcal{T} f)(y)=\tilde{f}(y) .
$$

Vector fields are transformed here coordinatewise (as independent scalars). More precisely,

$$
\widetilde{v}_{j}(y)=v_{j}(x)=v_{j}\left(y^{\prime}, y_{3}+\eta\left(y^{\prime}\right)\right),
$$

where $j=1,2,3$. Conversely,

$$
v_{j}(x)=\widetilde{v}_{j}(y)=v_{j}\left(x^{\prime}, x_{3}-\eta\left(x^{\prime}\right)\right) .
$$

Given $x$, if $y=T x$ then $y^{\prime}=x^{\prime}$. Hence $\tilde{\eta}(y)=\eta(x)=\eta\left(x^{\prime}\right)=\eta\left(y^{\prime}\right)$, moreover $\partial \eta\left(x^{\prime}\right) / \partial x_{j}=\partial \eta\left(y^{\prime}\right) / \partial y_{j}$, and so on. In the following we identify the above functions and use the sole notation $\eta\left(y^{\prime}\right)$.

We set

$$
\begin{aligned}
\mathbb{V}\left(\Omega_{a}\right) & =\left\{v: v \in \mathbb{W}_{0}^{1, p}\left(\Omega_{a}\right), \operatorname{supp} v \subset I_{a}\right\}, \\
\mathbb{V}\left(Q_{a}\right) & =\left\{\widetilde{v}: \widetilde{v} \in \mathbb{W}_{0}^{1, p}\left(Q_{a}\right), \operatorname{supp} \tilde{v} \subset J_{a}\right\} .
\end{aligned}
$$

Clearly, if a test function $\phi(x)$ belongs to $\mathbb{V}\left(\Omega_{a}\right)$ the transformed function $\widetilde{\phi}(y)$ belongs to $\mathbb{V}\left(Q_{a}\right)$.

A main point in what follows is that

$$
\partial_{j} \eta(0)=0, \quad j=1,2,
$$


which holds since $\Pi$ is tangent to $\Gamma$ at $x_{0}$. The following result is a consequence of (5.7) together with the continuity of $\nabla \eta$ over $\Gamma$.

Lemma 5.1. Given a positive real $\epsilon_{0}$, there is an $a\left(\epsilon_{0}\right)>0$ such that if $a \leq a\left(\epsilon_{0}\right)$ then

$$
\mid\left(\nabla \eta\left(y^{\prime}\right) \mid<\epsilon_{0} \quad \text { for all } y^{\prime} \text { such that }\left|y^{\prime}\right|<a .\right.
$$

Moreover, a $\left(\epsilon_{0}\right)$ is independent of the point $x_{0}$.

Note that $a\left(\epsilon_{0}\right)$ depends on the $C^{1}\left(J_{a}\right)$ norm of $\eta$. Since $\Gamma$ is compact the desired independence holds.

From (5.4) it follows that, for each $y$,

$$
\widetilde{\partial_{k} \phi}=\partial_{k} \widetilde{\phi}-\left(\partial_{k} \eta\right)\left(y^{\prime}\right) \partial_{3} \widetilde{\phi}
$$

where $\partial_{k} \eta$ is calculated at $y^{\prime}$. Note that the first equation (5.9) holds for $k=3$ since $\partial_{3} \eta=0$. By iteration, the above formula may be extended to higher order derivatives (not used later):

$$
\mathcal{T}\left(\partial_{j k}^{2} \phi\right)=\partial_{j k}^{2} \tilde{\phi}-\left(\partial_{k} \eta\right) \partial_{j 3}^{2} \tilde{\phi}-\left(\partial_{j} \eta\right) \partial_{k 3}^{2} \tilde{\phi}+\left(\partial_{j} \eta\right)\left(\partial_{k} \eta\right) \partial_{3}^{2} \widetilde{\phi}-\left(\partial_{j k}^{2} \eta\right) \partial_{3} \tilde{\phi} .
$$

Remark 5.1. We want to emphasize that, basically, our regularity results will be proved in the following local form. Let $x_{0}$ and $\Omega_{a}$ be as above. If $(u, \pi) \in \mathbb{W}^{1, p}\left(\Omega_{a}\right) \times L^{p^{\prime}}\left(\Omega_{a}\right)$ satisfies (2.1) in the weak sense in $\Omega_{a}$ and satisfies (1.4) in $\Gamma_{a}$, then the regularity results hold in $\Omega_{r}$ for $r<a$ (for instance, for $r=a / 2$ ). We prove this local result by assuming that $a>0$ is sufficiently small. Our final value of $a$ is not necessarily equal to the initial one. As we proceed through the proof we may need to consider smaller values of $a$. However, we will show explicitly that each new (smaller) value of $a$ depends only on an upper bound of the $C^{2}\left(J_{a}\right)$ norm of $\eta$. In particular, a positive lower bound for $a$, independent of the point $x_{0}$, exists since $\Gamma$ is compact. This leads to the global result in the whole of $\Omega$.

\section{Translations and related properties}

In general we set

$$
\mathbb{T}_{\text {sym }}=\frac{1}{2}\left(\mathbb{T}+\mathbb{T}^{T}\right),
$$

where $\mathbb{T}$ denotes a generic tensor field, and $\mathbb{T}^{T}$ is its transpose. In particular, $\mathcal{D} u=$ $(\nabla u)_{\text {sym }}$.

We assume the reader is acquainted with the classical differential quotients (or translations) method. Nevertheless, we make some preliminary remarks. Roughly speaking, in order to prove regularity results for higher order derivatives of solutions with respect to a particular $x_{j}$ variable, this method requires that small translations of test functions in the $x_{j}$ (positive and negative) directions are still admissible test functions. This requirement immediately precludes the normal direction to the boundary. Actually, if $\Gamma_{a}$ is not flat near $x_{0}$, it precludes (in general) all the $x_{j}$ directions (this is obvious). However, if $\Gamma_{a}$ is flat, the tangential directions are admissible. In fact, if $\phi$ vanishes on $\Gamma_{a}$, small transla- 
tions of $\phi$ in any tangential direction are well defined in $\Omega_{a}$ and vanish on $\Gamma_{a}$ (actually, for a smaller value of the parameter $a$ ). This leads to the possibility of applying the differential quotients method with respect (roughly speaking!) to the tangent directions $x_{1}$ and $x_{2}$ at the point $x_{0}$, simply by making the change of variables introduced in the previous section and by doing translations with respect to the new tangent directions $y_{1}$ and $y_{2}$. This explains why, below, we deal with translations of $h_{j}$ in the $y_{j}$-directions, $j=1,2$. For notational convenience we consider the case $j=1$ and set $h=h_{1}$. All the results hold for $j=2$. We use the following convention:

$$
y+h=\left(y_{1}+h, y_{2}, y_{3}\right), \quad y^{\prime}+h=\left(y_{1}+h, y_{2}\right) .
$$

The amplitude $|h|$ of the translations is always assumed to be smaller than the distance from the support of $\widetilde{\phi}$ to the set $\left(\partial Q_{a}\right) \backslash \Lambda_{a}$.

The following remark concerns a fundamental point in our approach.

Remark 6.1. A test function $\phi(x)$ is transformed into a function $\widetilde{\phi}(y)$. Since in the following we make translations with respect to the $y$ variables we need to determine (and study the differential properties of) the particular test function $\phi_{h}(x)$ such that $\left(\widetilde{\phi}_{h}\right)(y)=$ $\widetilde{\phi}(y+h)$, for each test function $\phi(x)$ and each small amplitude $h$. This is the aim of this section.

The above functions $\phi_{h}(x)$ are called pseudo-translations.

Lemma 6.1. Let $\phi(x) \in \mathbb{V}\left(\Omega_{a}\right)$. Define $\phi_{h}$ by

$$
\phi_{h}(x)=\phi\left(x_{1}+h, x_{2}, x_{3}-\eta\left(x^{\prime}\right)+\eta\left(x^{\prime}+h\right)\right) .
$$

Then

$$
\widetilde{\phi}_{h}(y)=\widetilde{\phi}(y+h) .
$$

The verification is left to the reader.

Next we want to establish the transformation law for derivatives of the pseudo-translations $\phi_{h}(x)$. One has the following result.

Lemma 6.2. Let $\phi(x) \in \mathbb{V}\left(\Omega_{a}\right)$, let $\phi_{h}(x)$ be as in the previous lemma, and let $k \leq 3$ be fixed. Then

$$
\left(\widetilde{\partial_{k} \phi_{h}}\right)(y)=\left(\widetilde{\partial_{k} \phi}\right)(y+h)+\left(\widetilde{\partial_{3} \phi}\right)(y+h)\left[\left(\partial_{k} \eta\right)\left(y^{\prime}+h\right)-\left(\partial_{k} \eta\right)\left(y^{\prime}\right)\right] .
$$

If $k=3$ the second term on the right hand side vanishes identically.

Proof. From 6.2 it readily follows that

$$
\begin{aligned}
\left(\partial_{k} \phi_{h}\right)(x)= & \left(\partial_{k} \phi\right)\left(x^{\prime}+h, x_{3}+\eta\left(x^{\prime}+h\right)-\eta\left(x^{\prime}\right)\right) \\
& +\left(\partial_{n} \phi\right)\left(x^{\prime}+h, x_{3}+\eta\left(x^{\prime}+h\right)-\eta\left(x^{\prime}\right)\right)\left[\left(\partial_{k} \eta\right)\left(x^{\prime}+h\right)-\left(\partial_{k} \eta\right)\left(x^{\prime}\right)\right] .
\end{aligned}
$$

Note that the last term is not taken into account if $k=3$. By the definition of the "tilde" functions

$$
\left(\widetilde{\partial_{k} \phi_{h}}\right)(y)=\left(\partial_{k} \phi_{h}\right)\left(T^{-1} y\right)=\left(\partial_{k} \phi_{h}\right)(\bar{x})
$$


where

$$
\bar{x}=\left(\bar{x}_{1}, \bar{x}_{2}, \bar{x}_{3}\right)=\left(y^{\prime}, y_{3}+\eta\left(y^{\prime}\right)\right) .
$$

Hence from 6.5 with $x$ replaced by $\bar{x}$ we get an expression for $\left(\widetilde{D_{k} \phi_{h}}\right)(y)$ in terms of $\bar{x}$. By taking into account the definition of $\bar{x}$ we obtain

$$
\begin{aligned}
\left(\widetilde{\partial_{k} \phi_{h}}\right)(y)= & \left(\partial_{k} \phi\right)\left(y^{\prime}+h, y_{3}+\eta\left(y^{\prime}+h\right)\right) \\
& +\left(\partial_{3} \phi\right)\left(y^{\prime}+h, y_{3}+\eta\left(y^{\prime}+h\right)\right)\left[\left(\partial_{k} \eta\right)\left(y^{\prime}+h\right)-\left(\partial_{k} \eta\right)\left(y^{\prime}\right)\right] .
\end{aligned}
$$

Since $\left(y^{\prime}+h, y_{3}+\eta\left(y^{\prime}+h\right)\right)=T^{-1}(y+h)$ it follows that

$$
\left(\partial_{k} \phi\right)\left(y^{\prime}+h, y_{3}+\eta\left(y^{\prime}+h\right)\right)=\left(\widetilde{\partial_{k} \phi}\right)(y+h) .
$$

Consequently, 6.4 follows from 6.6.

By setting in general

$$
(\nabla \phi)_{i k}=\partial_{k} \phi_{i}
$$

it follows from 6.4 that

$$
\left(\widetilde{\nabla \phi_{h}}\right)(y)=(\widetilde{\nabla \phi})(y+h)+\left(\widetilde{\partial_{3} \phi}\right)(y+h) \otimes\left[(\nabla \eta)\left(y^{\prime}+h\right)-(\nabla \eta)\left(y^{\prime}\right)\right]
$$

where, since $\eta$ does not depend on the third. variable, we set

$$
\nabla \eta=\left(\partial_{1} \eta, \partial_{2} \eta\right) .
$$

In particular, since $\mathcal{D} u=(\nabla u)_{\text {sym }}($ recall $(6.1))$,

$$
\left(\widetilde{\mathcal{D} \phi_{h}}\right)(y)=(\widetilde{\mathcal{D} \phi})(y+h)+\left\{\left(\widetilde{\partial_{3} \phi}\right)(y+h) \otimes\left[(\nabla \eta)\left(y^{\prime}+h\right)-(\nabla \eta)\left(y^{\prime}\right)\right]\right\}_{\text {sym }} .
$$

Moreover,

$$
\left(\widetilde{\nabla \cdot \phi_{h}}\right)(y)=(\widetilde{\nabla \cdot \phi})(y+h)+\left(\widetilde{\partial_{3} \phi}\right)(y+h) \cdot\left[(\nabla \eta)\left(y^{\prime}+h\right)-(\nabla \eta)\left(y^{\prime}\right)\right] .
$$

Now we express the derivatives with respect to the $y$ variables of functions $\widetilde{\phi}(y)$ in terms of the transformations of the derivatives of the original functions $\phi(x)$.

Lemma 6.3. One has the following formulas:

$$
\begin{aligned}
& \left(\partial_{k} \widetilde{\phi}\right)(y)=\left(\widetilde{\partial_{k} \phi}\right)(y)+\left(\partial_{k} \eta\right)\left(y^{\prime}\right)\left(\widetilde{\partial_{3} \phi}\right)(y), \\
& \left(\widetilde{\partial_{k} \phi}\right)(y)=\left(\partial_{k} \widetilde{\phi}\right)(y)-\left(\partial_{k} \eta\right)\left(y^{\prime}\right)\left(\partial_{3} \widetilde{\phi}\right)(y) .
\end{aligned}
$$

If $k=3$ the second terms on the above right hand sides vanish identically.

Proof. Since $\widetilde{\phi}(y)=\phi\left(T^{-1} y\right)$ it follows that

$$
\left(\partial_{k} \widetilde{\phi}\right)(y)=\left(\partial_{k} \phi\right)\left(T^{-1} y\right)+\left(\partial_{3} \phi\right)\left(T^{-1} y\right)\left(\partial_{k} \eta\right)\left(y^{\prime}\right)=\left(\widetilde{\partial_{k} \phi}\right)(y)+\left(\partial_{k} \eta\right)\left(y^{\prime}\right)\left(\widetilde{\partial_{3} \phi}\right)(y) .
$$

Note that $\partial_{3} \widetilde{\phi}=\widetilde{\partial_{3} \phi}$.

From the above lemma it follows that

$$
\begin{aligned}
(\widetilde{\nabla \phi})(y) & =(\nabla \widetilde{\phi})(y)-(\nabla \eta)\left(y^{\prime}\right) \otimes\left(\partial_{3} \tilde{\phi}\right)(y), \\
(\widetilde{\nabla \cdot \phi})(y) & =(\nabla \cdot \widetilde{\phi})(y)-(\nabla \eta)\left(y^{\prime}\right) \cdot\left(\partial_{3} \widetilde{\phi}\right)(y) .
\end{aligned}
$$


Lemma 6.4. Given an $\left.\epsilon_{0} \in\right] 0,1\left[\right.$ there is an $a\left(\epsilon_{0}\right)>0$ such that if $a \leq a\left(\epsilon_{0}\right)$ then

$$
|(\nabla \widetilde{\phi})(y)-(\widetilde{\nabla \phi})(y)| \leq \epsilon_{0}\left|\left(\widetilde{\partial_{3} \phi}\right)(y)\right|, \quad \forall y \in Q_{a} .
$$

The same result holds if we replace y by $y-h$. Clearly we may replace $\nabla$ by $\mathcal{D}$.

Proof. From 6.10 one shows that the left hand side of 6.14 is bounded by $\left|\nabla \eta\left(y^{\prime}\right)\right|\left|\left(\widetilde{\partial_{3} \phi}\right)(y)\right|$. Since $\nabla \eta(0)=0$ it follows that $\left|\nabla \eta\left(y^{\prime}\right)\right| \leq \epsilon_{0}$ in a sufficiently small neighborhood of $x_{0}$.

Remark 6.2. Note that the identity $\nabla \phi(x)=(\widetilde{\nabla \phi})(y)$ together with 6.14) leads to a "pointwise equivalence" between $|\nabla \phi(x)|,|(\widetilde{\nabla \phi})(y)|$ and $|(\nabla \widetilde{\phi})(y)|$. In particular, the $L^{q}$-norms of these quantities are equivalent.

Lemma 6.5. Given an $\left.\epsilon_{0} \in\right] 0,1\left[\right.$ there is an $a\left(\epsilon_{0}\right)>0$ such that if $a \leq a\left(\epsilon_{0}\right)$ then

$$
\begin{aligned}
& \mid((\widetilde{\nabla \phi})(y)-(\widetilde{\nabla \phi})(y-h))-((\nabla \widetilde{\phi})(y)-(\nabla \widetilde{\phi})(y-h)) \mid \\
& \leq \epsilon_{0}\left|\left(\partial_{3} \widetilde{\phi}\right)(y)-\left(\partial_{3} \widetilde{\phi}\right)(y-h)\right|+|h|\|\eta\|_{C^{2}\left(Q_{a}\right)}\left|\left(\partial_{3} \widetilde{\phi}\right)(y-h)\right| .
\end{aligned}
$$

Proof. From 6.11 one has

$$
\begin{aligned}
& ((\widetilde{\nabla \phi})(y)-(\widetilde{\nabla \phi})(y-h))-((\nabla \widetilde{\phi})(y)-(\nabla \widetilde{\phi})(y-h)) \\
& \quad=-\nabla \eta\left(y^{\prime}\right) \otimes\left(\left(\partial_{3} \widetilde{\phi}\right)(y)-\left(\partial_{3} \widetilde{\phi}\right)(y-h)\right)-\left(\nabla \eta\left(y^{\prime}\right)-\nabla \eta\left(y^{\prime}-h\right)\right) \otimes\left(\partial_{3} \widetilde{\phi}\right)(y-h) .
\end{aligned}
$$

Hence, in a sufficiently small neighborhood of $x_{0}, 6.15$ holds.

\section{Estimates for some second order derivatives of the velocity in terms of the pressure}

Further notation. For convenience, in the following $C$ denotes positive constants which are bounded from above provided that the quantities $\|\nabla \eta\|_{C^{1}\left(\Lambda_{a}\right)}$ and $\|\nabla \theta\|_{C^{1}\left(\Omega_{a}\right)}$ are bounded from above. The truncation function $\theta$ is defined below. In particular, this means that

$$
C=C\left(\|\nabla \eta\|_{C^{1}\left(\Lambda_{a}\right)},\|\nabla \theta\|_{C^{1}\left(\Omega_{a}\right)}\right)
$$

Nevertheless, for the reader's convenience (and for completeness) we often write the explicit dependence on the above quantities before including them in a constant of type $C$. Multiplicative constants of type $c$ will be incorporated in $C$.

In the following, in the absence of an explicit indication, tilde-functions inside integrals are calculated at the generic point $y$. Compare equations (7.1) and (7.2). Moreover, in the absence of an explicit indication, norms of functions of the $x$ variable concern the domain $\Omega_{a}$ and norms of tilde-functions concern the domain $Q_{a}$.

From (4.2), by making the change of variables $x \mapsto T x=y$, it follows that

$$
\begin{aligned}
\frac{v_{0}}{2} \int \widetilde{\nabla u} \cdot \widetilde{\nabla \phi} d y+v_{1} \int|\widetilde{\mathcal{D} u}|^{p-2} \widetilde{\mathcal{D} u} \cdot \widetilde{\mathcal{D} \phi} d y \\
\quad-\int \widetilde{\pi}(\widetilde{\nabla \cdot \phi}) d y+\int(\widetilde{\nabla \cdot u}) \widetilde{\psi} d y=\int \tilde{f} \cdot \widetilde{\phi} d y
\end{aligned}
$$


for each $\widetilde{\phi} \in \mathbb{V}\left(Q_{a}\right)$ and each $\widetilde{\psi} \in L^{p}\left(Q_{a}\right)$. Recall that the Jacobian determinant of the $T$-transform is equal to one.

Next we consider the equation (7.1) with $\phi$ and $\psi$ replaced by the admissible test functions $\phi_{h}$ and $\psi_{h}$ respectively (recall (6.2)). Then by the change of variables $y \mapsto y-h$ we show that

$$
\begin{array}{r}
\frac{\nu_{0}}{2} \int \widetilde{\nabla u}(y-h): \widetilde{\nabla \phi_{h}}(y-h) d y+v_{1} \int|\widetilde{\mathcal{D} u}(y-h)|^{p-2} \widetilde{\mathcal{D} u}(y-h): \widetilde{\mathcal{D} \phi_{h}}(y-h) d y \\
-\int \tilde{\pi}(y-h)\left(\widetilde{\nabla \cdot \phi_{h}}(y-h)\right) d y+\int(\widetilde{\nabla \cdot u}(y-h)) \widetilde{\psi_{h}}(y-h) d y \\
=\int \tilde{f}(y-h) \cdot \widetilde{\phi}_{h}(y-h) d y
\end{array}
$$

for each $\widetilde{\phi} \in \mathbb{V}\left(Q_{a}\right)$ and each $\widetilde{\psi} \in L^{p}\left(Q_{a}\right)$.

By appealing to 6.3, (6.4), 6.7), 6.8) and 6.9) we may write (7.2) in the form

$$
\begin{aligned}
& \frac{\nu_{0}}{2} \int \widetilde{\nabla u}(y-h): \widetilde{\nabla \phi}(y) d y+\frac{\nu_{0}}{2} \int \widetilde{\nabla u}(y-h):\left[\left(\widetilde{\partial_{3} \phi}\right)(y) \otimes\left[(\nabla \eta)\left(y^{\prime}\right)-(\nabla \eta)\left(y^{\prime}-h\right)\right]\right] d y \\
& +v_{1} \int|\widetilde{\mathcal{D} u}(y-h)|^{p-2} \widetilde{\mathcal{D} u}(y-h): \widetilde{\mathcal{D} \phi}(y) d y \\
& +v_{1} \int|\widetilde{\mathcal{D} u}(y-h)|^{p-2} \widetilde{\mathcal{D} u}(y-h):\left[\left(\widetilde{\partial_{3} \phi}\right)(y) \otimes\left[(\nabla \eta)\left(y^{\prime}\right)-(\nabla \eta)\left(y^{\prime}-h\right)\right]\right]_{\mathrm{sym}} d y \\
& -\int \tilde{\pi}(y-h) \widetilde{(\nabla \cdot \phi)}(y) d y-\int \tilde{\pi}(y-h)\left(\widetilde{\partial_{3} \phi}\right)(y) \cdot\left[(\nabla \eta)\left(y^{\prime}\right)-(\nabla \eta)\left(y^{\prime}-h\right)\right] d y \\
& +\int \widetilde{(\nabla \cdot u)}(y-h) \widetilde{\psi}(y) d y=\int \tilde{f}(y-h) \cdot \tilde{\phi}(y) d y .
\end{aligned}
$$

Finally, by subtracting (7.1) and (7.3) we get

$$
\begin{aligned}
& \frac{\nu_{0}}{2} \int(\widetilde{\nabla u}(y)-\widetilde{\nabla u}(y-h)): \widetilde{\nabla \phi}(y) d y \\
& \quad+v_{1} \int\left(|\widetilde{\mathcal{D} u}(y)|^{p-2} \widetilde{\mathcal{D} u}(y)-|\widetilde{\mathcal{D} u}(y-h)|^{p-2} \widetilde{\mathcal{D} u}(y-h)\right): \widetilde{(\mathcal{D} \phi)}(y) d y \\
& \left.\quad-\int(\widetilde{\pi}(y)-\widetilde{\pi}(y-h))(\widetilde{\nabla \cdot \phi})(y) d y+\int(\widetilde{\nabla \cdot u})(y)-\widetilde{(\nabla \cdot u)}(y-h)\right) \widetilde{\psi}(y) d y \\
& =-\int \widetilde{f}(y) \cdot(\widetilde{\phi}(y+h)-\widetilde{\phi}(y)) d y \\
& \quad+\frac{\nu_{0}}{2} \int \widetilde{\nabla v}(y-h):\left[\left(\widetilde{\partial_{3} \phi}\right)(y) \otimes\left[(\nabla \eta)\left(y^{\prime}\right)-(\nabla \eta)\left(y^{\prime}-h\right)\right]\right] d y \\
& \quad+v_{1} \int|\widetilde{\mathcal{D} u}(y-h)|^{p-2} \widetilde{\mathcal{D} u}(y-h):\left[\left(\widetilde{\partial_{3} \phi}\right)(y) \otimes\left[(\nabla \eta)\left(y^{\prime}\right)-(\nabla \eta)\left(y^{\prime}-h\right)\right]\right]_{\mathrm{sym}} d y \\
& \quad-\int \widetilde{\pi}(y-h)\left(\widetilde{\partial_{3} \phi}\right)(y) \cdot\left[(\nabla \eta)\left(y^{\prime}\right)-(\nabla \eta)\left(y^{\prime}-h\right)\right] d y .
\end{aligned}
$$


Remark 7.1. A fundamental remark: Now we would like to replace in 7.4$) \widetilde{\nabla \phi}(y)$ with $\widetilde{\nabla u}(y)-\widetilde{\widetilde{\nabla} u}(y-h)$, and consequently $\widetilde{\mathcal{D} \phi}(y)$ with $\widetilde{\mathcal{D} u}(y)-\widetilde{\mathcal{D} u}(y-h)$. Unfortunately, this is not allowed since $\widetilde{\nabla u}(y-h)$ is not the transformation of the gradient of an $x$-test function. However, our goal will be obtained "up to a perturbation term" by setting in equation (7.4)

$$
\phi(x)=\left(u(x)-u_{-h}(x)\right) \theta^{2}(x),
$$

where $\theta$ is an arbitrary regular real function such that

$$
\operatorname{supp} \theta \subset I_{a}
$$

To fix ideas also assume from now on that $0<\theta(x) \leq 1$. Note that $\tilde{\theta}^{2}=(\tilde{\theta})^{2}$ and $\widetilde{\nabla \theta^{2}}=2 \widetilde{\theta} \widetilde{\nabla \theta}$. Clearly

$$
\widetilde{\phi}(y)=(\widetilde{u}(y)-\widetilde{u}(y-h))(\widetilde{\theta})^{2}(y) .
$$

Lemma 7.1. Let $\phi(x)$ be the admissible test function given by 7.5 . Then the $y$-transforms of $\nabla \phi(x), \mathcal{D} \phi(x), \partial_{3} \phi(x)$ and $\nabla \cdot \phi(x)$ are respectively given by (7.7), (7.8), (7.9) and 7.10 ) below.

Proof. By taking the gradient of both sides of (7.5), by passing from the $x$ to the $y$ variables and by appealing to 6.7 it readily follows that

$$
\begin{aligned}
\widetilde{\nabla \phi}(y)= & (\widetilde{\nabla u}(y)-\widetilde{\nabla u}(y-h))(\widetilde{\theta})^{2}(y) \\
& +\left(\widetilde{\partial_{3} u}\right)(y-h) \otimes\left[(\nabla \eta)\left(y^{\prime}\right)-(\nabla \eta)\left(y^{\prime}-h\right)\right](\widetilde{\theta})^{2}(y) \\
& +2 \widetilde{\theta}(y)(\widetilde{u}(y)-\widetilde{u}(y-h)) \otimes \widetilde{\nabla \theta}(y) .
\end{aligned}
$$

In particular, one has

$$
\begin{aligned}
\widetilde{\mathcal{D} \phi}(y)= & (\widetilde{\mathcal{D}} u(y)-\widetilde{\mathcal{D}} u(y-h))(\widetilde{\theta})^{2}(y) \\
& +\left\{\left(\widetilde{\partial_{3} u}\right)(y-h) \otimes\left[(\nabla \eta)\left(y^{\prime}\right)-(\nabla \eta)\left(y^{\prime}-h\right)\right](\widetilde{\theta})^{2}(y)\right\}_{\mathrm{sym}} \\
& +2 \widetilde{\theta}(y)\{(\tilde{u}(y)-\widetilde{u}(y-h)) \otimes \widetilde{\nabla \theta}(y)\}_{\mathrm{sym}},
\end{aligned}
$$

and also (since $\left.\partial_{3} \eta=0\right)$,

$$
\widetilde{\partial_{3} \phi}(y)=\left(\widetilde{\partial_{3} u}(y)-\widetilde{\partial_{3} u}(y-h)\right)(\widetilde{\theta})^{2}(y)+2 \widetilde{\theta}(y)(\widetilde{u}(y)-\widetilde{u}(y-h)) \widetilde{\partial_{3} \theta}(y) .
$$

Similarly, from 6.3 and 6.9 it readily follows that

$$
\begin{aligned}
\widetilde{\nabla \cdot \phi}(y)= & (\widetilde{\nabla \cdot u}(y)-\widetilde{\nabla \cdot u}(y-h))(\widetilde{\theta})^{2}(y) \\
& +\left(\widetilde{\partial_{3} u}\right)(y-h) \cdot\left[(\nabla \eta)\left(y^{\prime}\right)-(\nabla \eta)\left(y^{\prime}-h\right)\right](\widetilde{\theta})^{2}(y) \\
& +2 \widetilde{\theta}(y)(\widetilde{u}(y)-\widetilde{u}(y-h)) \cdot \widetilde{\nabla \theta}(y) .
\end{aligned}
$$


On the other hand, by setting

$$
\psi(x)=\left(\pi(x)-\pi_{-h}(x)\right) \theta^{2}(x),
$$

it follows that

$$
\widetilde{\psi}(y)=(\tilde{\pi}(y)-\tilde{\pi}(y-h))(\widetilde{\theta})^{2}(y) .
$$

Next we replace in (7.4) the test functions $\phi$ and $\psi$ by the expressions in (7.5) and (7.11). We start by estimating each of the terms that appear in (7.4). In order to treat the second integral on the left hand side of (7.4) we appeal to the following well known result.

Let $U, V$ be two arbitrary vectors in $\mathbb{R}^{N}, N \geq 1$, and $p \geq 2$. Then

$$
\begin{aligned}
\left(|U|^{p-2} U-|V|^{p-2} V\right) \cdot(U-V) & \geq \frac{1}{2}\left(|U|^{p-2}+|V|^{p-2}\right)|U-V|^{2}, \\
\left.|| U\right|^{p-2} U-|V|^{p-2} V \mid & \leq \frac{p-1}{2}\left(|U|^{p-2}+|V|^{p-2}\right)|U-V| .
\end{aligned}
$$

Proposition 7.1. Let $\widetilde{\phi}(y)$ be given by (7.5). Then

$$
\begin{gathered}
v_{1} \int\left(|\widetilde{\mathcal{D}} u(y)|^{p-2} \widetilde{\mathcal{D} u}(y)-|\widetilde{\mathcal{D} u}(y-h)|^{p-2} \widetilde{\mathcal{D} u}(y-h)\right): \widetilde{\mathcal{D} \phi}(y) d y \\
\geq \frac{v_{1}}{4} \int\left(|(\widetilde{\mathcal{D} u})(y)|^{p-2}+|(\widetilde{\mathcal{D} u})(y-h)|^{p-2}\right)|(\widetilde{\mathcal{D} u})(y)-(\widetilde{\mathcal{D} u})(y-h)|^{2}(\widetilde{\theta})^{2}(y) d y \\
-C\|\nabla \widetilde{u}\|_{p}^{p} h^{2} .
\end{gathered}
$$

Proof. For convenience, denote by $S_{1}$ the left hand side of (7.14). By (7.8) one has

$$
\begin{aligned}
& S_{1}= v_{1} \int\left(|\widetilde{\mathcal{D} u}(y)|^{p-2} \widetilde{\mathcal{D} u}(y)-|\widetilde{\mathcal{D} u}(y-h)|^{p-2} \widetilde{\mathcal{D} u}(y-h)\right) \\
& \cdot(\widetilde{\mathcal{D} u}(y)-\widetilde{\mathcal{D} u}(y-h))(\widetilde{\theta})^{2}(y) d y \\
&+v_{1} \int\left(|\widetilde{\mathcal{D} u}(y)|^{p-2} \widetilde{\mathcal{D} u}(y)-|\widetilde{\mathcal{D} u}(y-h)|^{p-2} \widetilde{\mathcal{D} u}(y-h)\right) \\
& \cdot\left\{\left(\widetilde{\partial_{3} u}\right)(y-h) \otimes\left[(\nabla h)\left(y^{\prime}\right)-(\nabla h)\left(y^{\prime}-h\right)\right]\right\}_{\mathrm{sym}}(\widetilde{\theta})^{2}(y) d y \\
&+2 v_{1} \int\left(|\widetilde{\mathcal{D} u}(y)|^{p-2} \widetilde{\mathcal{D} u}(y)-|\widetilde{\mathcal{D} u}(y-h)|^{p-2} \widetilde{\mathcal{D} u}(y-h)\right) \\
& \cdot\{(\widetilde{u}(y)-\widetilde{u}(y-h)) \otimes \widetilde{\nabla \theta}(y)\}_{\mathrm{sym}} \widetilde{\theta}(y) d y .
\end{aligned}
$$

From 7.13 it follows that

$$
\begin{aligned}
& S_{1} \geq \frac{v_{1}}{2} \int\left(|\widetilde{\mathcal{D} u}(y)|^{p-2}+|\widetilde{\mathcal{D} u}(y-h)|^{p-2}\right)|\widetilde{\mathcal{D} u}(y)-\widetilde{\mathcal{D} u}(y-h)|^{2}(\widetilde{\theta})^{2}(y) d y \\
&- \frac{v_{1}}{2}(p-1) \int\left(|\widetilde{\mathcal{D} u}(y)|^{p-2}+|\widetilde{\mathcal{D} u}(y-h)|^{p-2}\right)|\widetilde{\mathcal{D} u}(y)-\widetilde{\mathcal{D} u}(y-h)| \\
& \cdot|(\widetilde{\partial 3} u)(y-h)|\left|(\nabla \eta)\left(y^{\prime}\right)-(\nabla \eta)\left(y^{\prime}-h\right)\right|(\widetilde{\theta})^{2}(y) d y \\
&-v_{1}(p-1) \int\left(|\widetilde{\mathcal{D} u}(y)|^{p-2}+|\widetilde{\mathcal{D} u}(y-h)|^{p-2}\right) \\
& \cdot|\widetilde{\mathcal{D} u}(y)-\widetilde{\mathcal{D} u}(y-h)| \widetilde{\theta}(y)|\widetilde{u}(y)-\widetilde{u}(y-h)||\widetilde{\nabla \theta}(y)| d y .
\end{aligned}
$$


By appealing to the Cauchy-Schwarz inequality one easily shows that

$$
\begin{aligned}
& S_{1} \geq \frac{v_{1}}{4} \int\left(|\widetilde{\mathcal{D} u}(y)|^{p-2}+|\widetilde{\mathcal{D} u}(y-h)|^{p-2}\right)|\widetilde{\mathcal{D} u}(y)-\widetilde{\mathcal{D} u}(y-h)|^{2}(\widetilde{\theta})^{2}(y) d y \\
&-\frac{v_{1}}{2}(p-1)^{2} \int\left(|\widetilde{\mathcal{D} u}(y)|^{p-2}+|\widetilde{\mathcal{D} u}(y-h)|^{p-2}\right)\left|\left(\widetilde{\partial_{3} u}\right)(y-h)\right|^{2} \\
& \cdot\left|(\nabla \eta)\left(y^{\prime}\right)-(\nabla \eta)\left(y^{\prime}-h\right)\right|^{2}(\widetilde{\theta})^{2}(y) d y \\
&-2 v_{1}(p-1)^{2} \int\left(|\widetilde{\mathcal{D} u}(y)|^{p-2}+|\widetilde{\mathcal{D} u}(y-h)|^{p-2}\right)|\widetilde{u}(y)-\widetilde{u}(y-h)|^{2}|\widetilde{\nabla \theta}(y)|^{2} d y .
\end{aligned}
$$

The last two integrals are bounded by

$$
c h^{2}\left(\left\|D^{2} \eta\right\|_{\infty}^{2}+\left\|D^{2} \theta\right\|_{\infty}^{2}\right)\|\widetilde{\nabla u}(y)\|_{p}^{p} .
$$

Next we estimate the third integral on the right hand side of (7.4).

Proposition 7.2. Let $\widetilde{\phi}(y)$ be given by 7.5 . Then

$$
\begin{aligned}
& \left.v_{1}\left|\int\right| \widetilde{\mathcal{D} u}(y-h)\right|^{p-2} \widetilde{\mathcal{D} u}(y-h):\left[\left(\widetilde{\partial_{3} \phi}\right)(y) \otimes\left[(\nabla \eta)\left(y^{\prime}\right)-(\nabla \eta)\left(y^{\prime}-h\right)\right]\right]_{\mathrm{sym}} d y \mid \\
& \leq \frac{v}{8} \int\left(|\widetilde{\mathcal{D} u}(y)|^{p-2}+|\widetilde{\mathcal{D} u}(y-h)|^{p-2}\right)|\widetilde{\mathcal{D} u}(y)-\widetilde{\mathcal{D} u}(y-h)|^{2}(\widetilde{\theta})^{2}(y) d y+C h^{2}\|\nabla \widetilde{u}\|_{p}^{p} .
\end{aligned}
$$

Proof. Denote by $S$ the integral on the left hand side of (7.18). By (7.8) one has

$$
\begin{aligned}
v_{1} S= & v_{1} \int|\widetilde{\mathcal{D} u}(y-h)|^{p-2} \widetilde{\mathcal{D} u}(y-h) \\
& \cdot\left[\left(\widetilde{\partial_{3} u}(y)-\widetilde{\partial_{3} u}(y-h)\right)(\widetilde{\theta})^{2}(y) \otimes\left[(\nabla \eta)\left(y^{\prime}\right)-(\nabla \eta)\left(y^{\prime}-h\right)\right]\right]_{\mathrm{sym}} d y \\
+ & 2 v_{1} \int|\widetilde{\mathcal{D} u}(y-h)|^{p-2} \widetilde{\mathcal{D} u}(y-h) \\
& \cdot\left[(\widetilde{u}(y)-\widetilde{u}(y-h)) \widetilde{\partial_{3} \theta}(y) \otimes\left[(\nabla \eta)\left(y^{\prime}\right)-(\nabla \eta)\left(y^{\prime}-h\right)\right]\right]_{\mathrm{sym}} \widetilde{\theta}(y) d y .
\end{aligned}
$$

The second integral on the right hand side of 7.19 is easily seen to be bounded by

$$
c\|\nabla \theta\|_{\infty}\left\|D^{2} \eta\right\|_{\infty}\|\nabla \tilde{u}\|_{p}^{p} h^{2},
$$

hence bounded by the last term on the right hand side of (7.18).

Denote by $I_{1}$ the first integral on the right hand side of $(7.19)$. By splitting it into two integrals, the first including $\widetilde{\partial_{3} u}(y)$ and the second including $\widetilde{\partial_{3} u}(y-h)$; by appealing to the change of variables $y_{1}-h \mapsto y_{1}$ in the second integral; and, finally, by splitting this last integral in a convenient and obvious way, we get 


$$
\begin{aligned}
I_{1}= & \int|\widetilde{\mathcal{D} u}(y-h)|^{p-2} \widetilde{\mathcal{D} u}(y-h):\left[\widetilde{\partial_{3} u}(y)(\widetilde{\theta})^{2}(y) \otimes\left[(\nabla \eta)\left(y^{\prime}\right)-(\nabla \eta)\left(y^{\prime}-h\right)\right]\right]_{\mathrm{sym}} d y \\
& -\int|\widetilde{\mathcal{D} u}(y)|^{p-2} \widetilde{\mathcal{D} u}(y):\left[\widetilde{\partial_{3} u}(y)(\widetilde{\theta})^{2}(y) \otimes\left[(\nabla \eta)\left(y^{\prime}\right)-(\nabla \eta)\left(y^{\prime}-h\right)\right]\right]_{\mathrm{sym}} d y \\
& -\int|\widetilde{\mathcal{D} u}(y)|^{p-2} \widetilde{\mathcal{D} u}(y):\left\{\left[\widetilde{\partial_{3} u}(y)(\widetilde{\theta})^{2}(y+h) \otimes\left[(\nabla \eta)\left(y^{\prime}+h\right)-(\nabla \eta)\left(y^{\prime}\right)\right]\right]_{\mathrm{sym}}\right. \\
& \left.-\left[\widetilde{\partial_{3} u}(y)(\widetilde{\theta})^{2}(y) \otimes\left[(\nabla \eta)\left(y^{\prime}\right)-(\nabla \eta)\left(y^{\prime}-h\right)\right]\right]_{\mathrm{sym}}\right\} d y .
\end{aligned}
$$

The last integral on the right hand side of $(7.20)$ is bounded by $C\|\nabla \eta\|_{\infty}\|\nabla \widetilde{u}\|_{p}^{p} h^{2}$, hence by the last term on the right hand side of (7.18). It remains to estimate the absolute value of the difference of the first two integrals on the right hand side of 7.20 . By appealing to 7.13 one shows that this absolute value is bounded by

$$
\begin{aligned}
& \frac{p-1}{2} \int\left(|\widetilde{\mathcal{D} u}(y)|^{p-2}+|\widetilde{\mathcal{D} u}(y-h)|^{p-2}\right) \\
& \cdot|\widetilde{\mathcal{D} u}(y)-\widetilde{\mathcal{D} u}(y-h)|\left|\widetilde{\partial_{3} u}(y)\right|(\widetilde{\theta})^{2}(y)\left|(\nabla \eta)\left(y^{\prime}\right)-(\nabla \eta)\left(y^{\prime}-h\right)\right| d y .
\end{aligned}
$$

In turn, this quantity is bounded by

$$
\begin{aligned}
& \frac{1}{8} \int\left(|\widetilde{\mathcal{D} u}(y)|^{p-2}+|\widetilde{\mathcal{D} u}(y-h)|^{p-2}\right)|\widetilde{\mathcal{D} u}(y)-\widetilde{\mathcal{D} u}(y-h)|^{2}(\widetilde{\theta})^{2}(y) d y \\
& +C \int\left|\widetilde{\partial_{3} u}(y)\right|^{2}\left|(\nabla \eta)\left(y^{\prime}\right)-(\nabla \eta)\left(y^{\prime}-h\right)\right|^{2}\left(|\widetilde{\mathcal{D} u}(y)|^{p-2}+|\widetilde{\mathcal{D} u}(y-h)|^{p-2}\right) \mid(\widetilde{\theta})^{2}(y) d y .
\end{aligned}
$$

As the last integral is bounded by $C\left\|D^{2} \eta\right\|_{\infty}^{2}\|\nabla \widetilde{u}\|_{p}^{p} h_{1}^{2}$, the estimate (7.18) follows.

From (7.14) and 7.18) we get the following result.

Proposition 7.3. Denote by $\mathcal{S}\left(v_{1}\right)$ the difference between the two $v_{1}$ terms that appear in equation 7.4 and let $\widetilde{\phi}(y)$ be given by 7.5 . Then

$$
\begin{aligned}
\mathcal{S}\left(v_{1}\right) \geq & \bar{c} v_{1} \int\left(|(\widetilde{\mathcal{D} u})(y)|^{p-2}+|(\widetilde{\mathcal{D} u})(y-h)|^{p-2}\right)|(\widetilde{\mathcal{D} u})(y)-(\widetilde{\mathcal{D} u})(y-h)|^{2}(\widetilde{\theta})^{2}(y) d y \\
& -C\|\nabla u\|_{p}^{p} h^{2} .
\end{aligned}
$$

Next we consider the " $\nu_{0}$ " terms that appear in (7.4). A simplification of the above argument (alternatively, set $p=2$ in (7.21) ) leads to the following result.

Proposition 7.4. Denote by $\mathcal{S}\left(\nu_{0}\right)$ the difference between the two $v_{0}$ terms that appear in equation (7.4) and let $\widetilde{\phi}(y)$ be given by 7.5 . Then

$$
\mathcal{S}\left(\nu_{0}\right) \geq \bar{c} \nu_{0} \int|(\widetilde{\nabla u})(y)-(\widetilde{\nabla u})(y-h)|^{2}(\widetilde{\theta})^{2}(y) d y-C\|\nabla u\|_{2}^{2} h_{1}^{2} .
$$


Next we consider the $f$-term. A classical result shows that

$$
\left|\int \tilde{f}(y) \cdot(\tilde{\phi}(y+h)-\tilde{\phi}(y)) d y\right| \leq h\|\tilde{f}\|_{2}\|\nabla \widetilde{\phi}\|_{2} .
$$

Since $\widetilde{\phi}(y)$ is given by $(7.6$, straightforward calculations yield (recall that $0 \leq \theta(x) \leq 1$ )

$$
\begin{aligned}
& \left|\int \tilde{f}(y) \cdot(\tilde{\phi}(y+h)-\widetilde{\phi}(y)) d y\right| \leq h\|\tilde{f}\|_{2, Q_{a}}\left(\int|\nabla \tilde{u}(y)-\nabla \tilde{u}(y-h)|^{2}(\tilde{\theta})^{2}(y) d y\right)^{1 / 2} \\
& +h^{2}\|\tilde{f}\|_{2}\|\nabla \tilde{u}\|_{2}\left\|\nabla(\widetilde{\theta})^{2}\right\|_{\infty} .
\end{aligned}
$$

At this point we write the equation that follows from 7.4 by appealing to Propositions 7.4 and 7.3 , and to 7.24 . One has

$$
\begin{aligned}
& \bar{c} v_{0} \int|(\widetilde{\nabla u})(y)-(\widetilde{\nabla u})(y-h)|^{2}(\widetilde{\theta})^{2}(y) d y \\
&\left.+\left.\bar{c} \nu_{1} \int(\mid \widetilde{\mathcal{D} u})(y)\right|^{p-2}+|(\widetilde{\mathcal{D} u})(y-h)|^{p-2}\right)|(\widetilde{\mathcal{D} u})(y)-(\widetilde{\mathcal{D} u})(y-h)|^{2}(\widetilde{\theta})^{2}(y) d y \\
& \leq \int \widetilde{\pi}(y)\left(\widetilde{\partial_{3} u}\right)(y-h) \cdot\left[(\nabla \eta)\left(y^{\prime}\right)-(\nabla \eta)\left(y^{\prime}-h\right)\right](\widetilde{\theta})^{2}(y) d y \\
&+\int(\widetilde{\pi}(y)-\widetilde{\pi}(y-h)) \widetilde{\theta}(y)(\widetilde{u}(y)-\widetilde{u}(y-h)) \cdot \widetilde{\nabla \theta}(y) d y \\
&-\int \widetilde{\pi}(y-h)\left(\widetilde{\partial_{3} u}\right)(y) \cdot\left[(\nabla \eta)\left(y^{\prime}\right)-(\nabla \eta)\left(y^{\prime}-h\right)\right](\widetilde{\theta})^{2}(y) d y \\
&-\int \tilde{\pi}(y-h)(\widetilde{u}(y)-\widetilde{u}(y-h)) \widetilde{\partial_{3} \theta}(y) \cdot\left[(\nabla \eta)\left(y^{\prime}\right)-(\nabla \eta)\left(y^{\prime}-h\right)\right] \widetilde{\theta}(y) d y \\
&+C\left(\|\nabla u\|_{2}^{2}+\|\nabla u\|_{p}^{p}\right) h^{2}+c \frac{h^{2}}{v_{0}}\|\tilde{f}\|_{2}^{2}+h^{2}\|\widetilde{f}\|_{2}\|\nabla \widetilde{u}\|_{2}\left\|\nabla(\widetilde{\theta})^{2}\right\|_{\infty} \cdot \quad(7.25)
\end{aligned}
$$

By recalling, if necessary, 6.10 one easily shows that the fourth integral on the right hand side above is bounded by $C\|\nabla \theta\|_{\infty}\|\pi\|_{p^{\prime}}\|\nabla u\|_{p} h^{2}$. Similar estimates hold for the first and third integrals. The above arguments prove the following result.

Theorem 7.1. The following estimate holds:

$$
\begin{aligned}
\bar{c} \nu_{0} \int \mid(\widetilde{\nabla u})(y)-(\widetilde{\nabla u})\left(y-\left.h_{)}\right|^{2}(\widetilde{\theta})^{2}(y) d y\right. \\
\left.+\left.\bar{c} \nu_{1} \int(\mid \widetilde{\mathcal{D} u})(y)\right|^{p-2}+|(\widetilde{\mathcal{D} u})(y-h)|^{p-2}\right)|(\widetilde{\mathcal{D} u})(y)-(\widetilde{\mathcal{D} u})(y-h)|^{2}(\widetilde{\theta})^{2}(y) d y \\
\leq\left|\int(\widetilde{\pi}(y)-\widetilde{\pi}(y-h)) \widetilde{\theta}(y)(\widetilde{u}(y)-\widetilde{u}(y-h)) \cdot \widetilde{\nabla \theta}(y) d y\right| \\
+C\left(1+\|\nabla \theta\|_{\infty}^{2}\right)\left(\|\nabla u\|_{2}^{2}+\|\nabla u\|_{p}^{p}+\|\pi\|_{p^{\prime}}\|\nabla u\|_{p}\right) h^{2} \\
+c \frac{h^{2}}{v_{0}}\|\widetilde{f}\|_{2}^{2}+h^{2}\|\widetilde{f}\|_{2}\|\nabla \tilde{u}\|_{2}\left\|\nabla(\widetilde{\theta})^{2}\right\|_{\infty} .
\end{aligned}
$$


For convenience we define the nonnegative quantities $\widetilde{A}_{0}$ and $\widetilde{A}_{1}$ by

$$
\widetilde{A}_{0}^{2}=\int|(\widetilde{\nabla u})(y)-(\widetilde{\nabla u})(y-h)|^{2}(\widetilde{\theta})^{2} d y
$$

and

$$
\left.\widetilde{A}_{1}^{2}=\left.\int(\mid \widetilde{\mathcal{D} u})(y)\right|^{p-2}+|(\widetilde{\mathcal{D} u})(y-h)|^{p-2}\right)|(\widetilde{\mathcal{D} u})(y)-(\widetilde{\mathcal{D} u})(y-h)|^{2}(\widetilde{\theta})^{2} d y,
$$

and also

$$
U(u, \pi)=\|\nabla u\|_{p}^{2}+\|\nabla u\|_{p}^{p}+\|\pi\|_{p^{\prime}}^{p^{\prime}}
$$

Theorem 7.1 yields the following result.

Theorem 7.2. The following estimate holds:

$$
\begin{aligned}
& v_{0} \widetilde{A}_{0}^{2}+v_{1} \widetilde{A}_{1}^{2} \\
& \quad \leq C|h|\|\nabla \widetilde{u}\|_{p}\|(\tilde{\pi}(y)-\tilde{\pi}(y-h)) \tilde{\theta}(y)\|_{p^{\prime}}+C U(u, \pi) h^{2}+C\|f\|_{2}^{2} h^{2} .
\end{aligned}
$$

\section{Estimates for the tangential derivatives of pressure in terms of velocity}

In the following, $p$ denotes an exponent that lies in the interval

$$
2 \leq p \leq 3
$$

and $q$ an exponent that lies in the interval

$$
p \leq q \leq 6 .
$$

Actually, for a fixed $p$, it is sufficient that $q \leq q_{\infty}$ where

$$
q_{\infty}=3(4-p) \text {. }
$$

In general, for $1<r<3$ we define the Sobolev embedding exponent $r^{*}$ by the equation

$$
\frac{1}{r^{*}}=\frac{1}{r}-\frac{1}{3}
$$

Given $p$ and $q$ as above we define $r=r(q)$ by

$$
\frac{1}{r}=\frac{p-2}{2 q}+\frac{1}{2} .
$$

Note that

$$
\frac{1}{r^{\prime}}=\frac{q-p+2}{2 q} .
$$

In what follows we assume that $\nabla u \in L^{q}$. In particular, 4.77 holds. In this section we prove the following result. 
Theorem 8.1. For sufficiently small positive values of a (which are independent of the particular point $x_{0}$ ) one has

$$
\begin{aligned}
\|(\tilde{\pi}(y)-\tilde{\pi}(y-h)) \widetilde{\theta}\|_{r} \leq & c \widetilde{A}_{0}+c\|\nabla u\|_{q}^{(p-2) / 2} \widetilde{A}_{1} \\
& +C|h|\left(\|\nabla u\|_{2}+\|\nabla u\|_{(p-1) r}^{p-1}+\|\pi\|_{r}\right)+C|h|\|f\|_{2} .
\end{aligned}
$$

This result follows as a corollary of the crucial estimate stated in the following theorem.

Theorem 8.2. Given $\epsilon_{0}>0$ there is $a\left(\epsilon_{0}\right)>0$ (independent of the point $x_{0}$ ) such that for $a \leq a\left(\epsilon_{0}\right)$, one has

$$
\begin{aligned}
&\left|\int \nabla[(\tilde{\pi}(y)-\tilde{\pi}(y-h)) \widetilde{\theta}] \cdot \widetilde{\phi} d y\right| \\
& \leq c\left(\widetilde{A}_{0}+C|h|\|\nabla u\|_{2}\right)\|\nabla \widetilde{\phi}\|_{2}+c\|\nabla u\|_{q}^{(p-2) / 2} \widetilde{A_{1}}\|\nabla \widetilde{\phi}\|_{r^{\prime}} \\
&+\epsilon_{0}\|(\tilde{\pi}(y)-\tilde{\pi}(y-h)) \tilde{\theta}\|_{r}\|\nabla \widetilde{\phi}\|_{r^{\prime}} \\
&+C|h|\left(\|\nabla u\|_{2}+\|\nabla u\|_{(p-1) r}^{p-1}+\|\pi\|_{r}+\|f\|_{2}\right)\|\nabla \widetilde{\phi}\|_{r^{\prime}}
\end{aligned}
$$

for each $\widetilde{\phi} \in C_{0}^{2}\left(Q_{a}\right)$.

Proof of Theorem 8.1 We start proving Theorem 8.1 by assuming that 8.6 holds. This implies that $\nabla[(\tilde{\pi}(y)-\tilde{\pi}(y-h)) \tilde{\theta}] \in W^{-1, r}\left(Q_{a}\right)$ and

$$
\begin{aligned}
\|\nabla[(\tilde{\pi}(y)-\tilde{\pi}(y-h)) \tilde{\theta}]\|_{-1, r} \leq & \epsilon_{0}\|(\tilde{\pi}(y)-\tilde{\pi}(y-h)) \widetilde{\theta}\|_{r} \\
& +c \widetilde{A}_{0}+c\|\nabla u\|_{q}^{(p-2) / 2} \widetilde{A}_{1} \\
& +C|h|\left(\|\nabla u\|_{2}+\|\nabla u\|_{q}^{p-1}+\|\pi\|_{r}+\|f\|_{2}\right) .
\end{aligned}
$$

A main point here is that $\tilde{\theta}$ has compact support in $J_{a}$. To fix ideas the reader may assume, once and for all, that

$$
\operatorname{supp} \tilde{\theta} \subset \bar{Q}_{a / 2}
$$

and that the translation amplitude satisfies $|h|<a / 2$. Next, by appealing to a well known Nečas result (see Appendix 2), one shows that

$$
\begin{aligned}
\|(\tilde{\pi}(y)-\tilde{\pi}(y-h)) \tilde{\theta}\|_{r} \leq & \epsilon_{0}\|(\tilde{\pi}(y)-\tilde{\pi}(y-h)) \tilde{\theta}\|_{r} \\
& +c \widetilde{A}_{0}+c\|\nabla u\|_{q}^{(p-2) / 2} \widetilde{A}_{1} \\
& +C|h|\left(\|\nabla u\|_{2}+\|\nabla u\|_{q}^{p-1}+\|\pi\|_{r}+\|f\|_{2}\right) .
\end{aligned}
$$

This proves Theorem 8.1

The rest of this section is devoted to the proof of 8.6 . We start by proving the following lemma. 
Lemma 8.1. For each $\widetilde{\phi} \in C_{0}^{2}\left(Q_{a}\right)$ one has

$$
\begin{aligned}
& \left|\int \nabla[(\tilde{\pi}(y)-\tilde{\pi}(y-h)) \widetilde{\theta}] \cdot \widetilde{\phi} d y\right| \\
& \left.\leq v_{0} \mid \int(\widetilde{\nabla u}(y)-\widetilde{\nabla u}(y-h)): \widetilde{\nabla(\theta \phi}\right)(y) d y \mid \\
& \quad+v_{1}\left|\int\left(|\widetilde{\mathcal{D} u}(y)|^{p-2} \widetilde{\mathcal{D} u}(y)-|\widetilde{\mathcal{D} u}(y-h)|^{p-2} \widetilde{\mathcal{D} u}(y-h)\right): \widetilde{\nabla(\theta \phi)}(y) d y\right| \\
& \left.\quad+\epsilon_{0}\|(\widetilde{\pi}(y)-\widetilde{\pi}(y-h)) \widetilde{\theta}\|_{r}\|\nabla \widetilde{\phi}\|_{r^{\prime}}+\mid \int \widetilde{f} \cdot(\widetilde{\theta \phi})(y+h)-(\widetilde{\theta \phi})(y)\right) d y \mid \\
& \quad+C|h|\left(\|\nabla u\|_{2}+\|\nabla u\|_{(p-1) r}^{p-1}+\|\pi\|_{r}\right)\|\nabla \widetilde{\phi}\|_{r^{\prime}},
\end{aligned}
$$

where $\epsilon_{0}$ and a are chosen below.

Proof. From (7.4) with $\psi=0$ and $\phi$ replaced by $\theta \phi$ one easily gets

$$
\begin{aligned}
& \left.\int(\tilde{\pi}(y)-\tilde{\pi}(y-h))(\widetilde{\nabla \cdot(\theta \phi})\right)(y) d y \\
& \left.=\frac{\nu_{0}}{2} \int(\widetilde{\nabla u}(y)-\widetilde{\nabla u}(y-h)): \widetilde{\nabla(\theta \phi}\right)(y) d y \\
& \quad+v_{1} \int\left(|\widetilde{\mathcal{D} u}(y)|^{p-2} \widetilde{\mathcal{D} u}(y)-|\widetilde{\mathcal{D} u}(y-h)|^{p-2} \widetilde{\mathcal{D} u}(y-h)\right) \cdot \widetilde{\mathcal{D}(\theta \phi)}(y) d y \\
& \quad+\int \widetilde{f}(y) \cdot((\widetilde{\theta \phi})(y+h)-(\widetilde{\theta \phi})(y)) d y+c \mathcal{R},
\end{aligned}
$$

where $\mathcal{R}$ satisfies

$$
\begin{aligned}
|\mathcal{R}| \leq & v_{0}|h|\|\eta\|_{C^{2}}\|\widetilde{\nabla u}\|_{2}\left\|\widetilde{\partial_{3}(\theta \phi)}\right\|_{2} \\
& +v_{1}|h|\|\eta\|_{C^{2}}\|\widetilde{\nabla u}\|_{(p-1) r}^{p-1}\left\|\widetilde{\partial_{3}(\theta \phi)}\right\|_{r^{\prime}}+|h|\|\eta\|_{C^{2}}\|\pi\|_{r}\left\|\widetilde{\partial_{3}(\theta \phi)}\right\|_{r^{\prime}} .
\end{aligned}
$$

Since $\widetilde{\partial_{3}(\theta \phi)}=\widetilde{\theta} \partial_{3} \widetilde{\phi}+\widetilde{\phi} \widetilde{\partial_{3} \theta}$ (recall, in particular, 6.11 for $k=3$ ) it follows that

$$
\left\|\widetilde{\partial_{3}(\theta \phi)}\right\|_{r^{\prime}} \leq C\|\nabla \widetilde{\phi}\|_{r^{\prime}} .
$$

Hence, with a simplified notation,

$$
|\mathcal{R}| \leq C|h|\left(\|\nabla u\|_{2}+\|\nabla u\|_{(p-1) r}^{p-1}+\|\pi\|_{r}\right)\|\nabla \widetilde{\phi}\|_{r^{\prime}} .
$$

Note that $2 \leq r^{\prime}$.

On the other hand, by appealing to 6.13 , one shows that

$$
(\widetilde{\nabla \cdot(\theta \phi}))=\widetilde{\theta} \nabla \cdot \widetilde{\phi}+\widetilde{\phi} \cdot \widetilde{\nabla \theta}-\widetilde{\theta}(\nabla \eta) \cdot\left(\partial_{3} \widetilde{\phi}\right)-\left(\partial_{3} \widetilde{\theta}\right)(\nabla \eta) \cdot \widetilde{\phi} .
$$


Hence we may decompose the left hand side of 8.10 as

$$
\begin{aligned}
&\left.\int(\tilde{\pi}(y)-\tilde{\pi}(y-h))(\widetilde{\nabla \cdot(\theta \phi})\right) d y=\int[(\tilde{\pi}(y)-\tilde{\pi}(y-h)) \widetilde{\theta}](\nabla \cdot \tilde{\phi}) d y \\
&+\int(\tilde{\pi}(y)-\tilde{\pi}(y-h))(\widetilde{\nabla \theta}) \cdot \tilde{\phi} d y-\int[(\tilde{\pi}(y)-\tilde{\pi}(y-h)) \tilde{\theta}](\nabla \eta) \cdot\left(\partial_{3} \tilde{\phi}\right) d y \\
&-\int(\tilde{\pi}(y)-\tilde{\pi}(y-h))\left(\partial_{3} \tilde{\theta}\right)(\nabla \eta) \cdot \tilde{\phi} d y .
\end{aligned}
$$

By means of a suitable translation one shows that

$$
\begin{aligned}
\int(\tilde{\pi}(y)-\tilde{\pi}(y-h))(\widetilde{\nabla \theta}) \cdot \widetilde{\phi} d y & =-\int \tilde{\pi}(\widetilde{\phi}(y+h)-\widetilde{\phi}(y)) \cdot \widetilde{(\nabla \theta)} d y \\
& -\int \tilde{\pi} \widetilde{\phi}(y+h) \cdot(\widetilde{\nabla \theta}(y+h)-\widetilde{\nabla \theta}(y)) d y .
\end{aligned}
$$

Hence the second integral on the right hand side of 8.13 satisfies

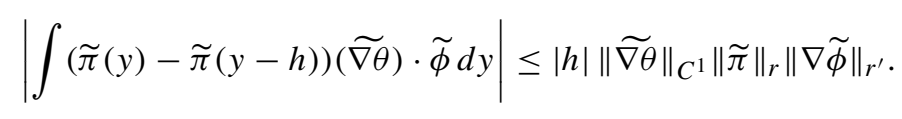

A similar device applied to the last integral on the right hand side of 8.13 shows that

$$
\left|\int(\tilde{\pi}(y)-\tilde{\pi}(y-h))\left(\partial_{3} \tilde{\theta}\right)(\nabla \eta) \cdot \widetilde{\phi} d y\right| \leq c|h|\|\nabla \eta\|_{C_{1}}\left\|\partial_{3} \tilde{\theta}\right\|_{C_{1}}\|\| \tilde{\pi}\left\|_{r}\right\| \nabla \tilde{\phi} \|_{r^{\prime}}
$$

On the other hand,

$$
\left|\int[(\tilde{\pi}(y)-\tilde{\pi}(y-h)) \widetilde{\theta}](\nabla \eta) \partial_{3} \widetilde{\phi} d y\right| \leq\|(\tilde{\pi}(y)-\widetilde{\pi}(y-h)) \widetilde{\theta}\|_{r}\|\nabla \eta\|_{C_{0}}\|\widetilde{\nabla \phi}\|_{r^{\prime}}
$$

From 8.13, 8.15, 8.16 and 8.17) it follows that

$$
\left.\int(\tilde{\pi}(y)-\tilde{\pi}(y-h))(\widetilde{\nabla \cdot(\theta \phi})\right) d y=-\int \nabla[(\tilde{\pi}(y)-\tilde{\pi}(y-h)) \tilde{\theta}] \cdot \tilde{\phi} d y+\mathcal{R}_{2}
$$

for each $\widetilde{\phi} \in C_{0}^{2}\left(Q_{a}\right)$, where $\mathcal{R}_{2}$ satisfies the estimate

$$
\left|\mathcal{R}_{2}\right| \leq C|h|\|\tilde{\pi}\|_{r}\|\nabla \tilde{\phi}\|_{r^{\prime}}+\epsilon_{0}\|(\tilde{\pi}(y)-\tilde{\pi}(y-h)) \tilde{\theta}\|_{r}\|\nabla \widetilde{\phi}\|_{r^{\prime}},
$$

for an arbitrarily small positive $\epsilon_{0}$, provided that $a \leq a\left(\epsilon_{0}\right)$. We have applied the fact that $\nabla \eta(0)=0$. From 8.18, 8.19) and (8.10, 8.12) the estimate 8.9) follows.

Next we estimate the $v_{0}$ and $v_{1}$ terms on the right hand side of 8.9 . We prove the following result. 
Lemma 8.2. The following estimates hold:

$$
\begin{aligned}
& v_{1} \mid \int\left(|\widetilde{\mathcal{D} u}(y)|^{p-2} \widetilde{\mathcal{D} u}(y)-\mid\right.\left.\left.\widetilde{\mathcal{D} u}(y-h)\right|^{p-2} \widetilde{\mathcal{D} u}(y-h)\right): \widetilde{\nabla(\theta \phi)} d y \mid \\
& \leq c\|\nabla u\|_{q}^{(p-2) / 2}\left(\widetilde{A_{1}}+C|h|\|\nabla u\|_{q}^{p / 2}\right)\|\nabla \widetilde{\phi}\|_{r^{\prime}}
\end{aligned}
$$

and

$$
\nu_{0}\left|\int(\widetilde{\nabla u}(y)-\widetilde{\nabla u}(y-h)): \widetilde{\nabla(\theta \phi)} d y\right| \leq c\left(\widetilde{A}_{0}+C|h|\|\nabla u\|_{2}\right)\|\nabla \widetilde{\phi}\|_{2} .
$$

Proof. From 6.12) it follows that

$$
\widetilde{\nabla(\theta \phi)}=\widetilde{\theta} \nabla \widetilde{\phi}-\tilde{\theta}\left[(\nabla \eta) \otimes \partial_{3} \widetilde{\phi}\right]+\widetilde{\phi} \otimes \widetilde{\nabla \theta}
$$

for each $y \in Q_{a}$. Moreover,

$$
\left|\widetilde{\theta} \nabla \widetilde{\phi}-\widetilde{\theta}\left[(\nabla \eta) \otimes \partial_{3} \widetilde{\phi}\right]\right| \leq C|\widetilde{\theta}||\nabla \widetilde{\phi}| .
$$

Hence, by 7.13, it follows that

$$
\begin{aligned}
& v_{1}\left|\int\left(|\widetilde{\mathcal{D} u}(y)|^{p-2} \widetilde{\mathcal{D} u}(y)-|\widetilde{\mathcal{D} u}(y-h)|^{p-2} \widetilde{\mathcal{D} u}(y-h)\right): \widetilde{\nabla(\theta \phi)} d y\right| \\
& \leq C v_{1} \int\left(|\widetilde{\mathcal{D} u}(y)|^{p-2}+|\widetilde{\mathcal{D} u}(y-h)|^{p-2}\right)|\widetilde{\mathcal{D} u}(y)-\widetilde{\mathcal{D} u}(y-h)||\widetilde{\theta}||\nabla \widetilde{\phi}| d y \\
& \quad+v_{1}\left|\int\left(|\widetilde{\mathcal{D} u}(y)|^{p-2} \widetilde{\mathcal{D} u}(y)-|\widetilde{\mathcal{D} u}(y-h)|^{p-2} \widetilde{\mathcal{D} u}(y-h)\right):(\widetilde{\phi} \otimes \widetilde{\nabla} \theta) d y\right| .
\end{aligned}
$$

Next,

$$
\begin{aligned}
& \int\left(|\widetilde{\mathcal{D} u}(y)|^{p-2}+|\widetilde{\mathcal{D} u}(y-h)|^{p-2}\right)|\widetilde{\mathcal{D} u}(y)-\widetilde{\mathcal{D} u}(y-h)||\widetilde{\theta}||\nabla \widetilde{\phi}| d y \\
& \leq c \int\left(|\widetilde{\mathcal{D} u}(y)|^{(p-2) / 2}+|\widetilde{\mathcal{D} u}(y-h)|^{(p-2) / 2}\right) \\
& \times\left[\left(|\widetilde{\mathcal{D} u}(y)|^{(p-2) / 2}+|\widetilde{\mathcal{D} u}(y-h)|^{(p-2) / 2}\right)|\widetilde{\mathcal{D} u}(y)-\widetilde{\mathcal{D} u}(y-h)||\widetilde{\theta}|\right]|\nabla \widetilde{\phi}| d y .
\end{aligned}
$$

By taking into account the definition of $r$ and by appealing to Hölder's inequality one shows that

$$
\begin{array}{r}
\int\left(|\widetilde{\mathcal{D} u}(y)|^{p-2}+|\widetilde{\mathcal{D} u}(y-h)|^{p-2}\right)|\widetilde{\mathcal{D} u}(y)-\widetilde{\mathcal{D} u}(y-h)||\widetilde{\theta}||\nabla \widetilde{\phi}| d y \\
\leq c\|\widetilde{\nabla u}\|_{q}^{(p-2) / 2} \widetilde{A_{1}}\|\nabla \widetilde{\phi}\|_{r^{\prime}}
\end{array}
$$

which is the desired estimate for the first integral on the right hand side of 8.23 . 
We could appeal to similar devices to obtain as well a useful estimate for the second integral there. However, the lack of $\widetilde{\theta}(y)$ in this integral would require some tricky arguments. We prefer to introduce a more elegant device. Denote by $I$ the integral in question. An obvious translation shows that

$$
\begin{aligned}
I= & \int|\widetilde{\mathcal{D} u}(y)|^{p-2} \widetilde{\mathcal{D} u}(y):(\widetilde{\phi}(y) \otimes \widetilde{\nabla \theta}(y)) d y \\
& -\int|\widetilde{\mathcal{D} u}(y)|^{p-2} \widetilde{\mathcal{D} u}(y):(\widetilde{\phi}(y+h) \otimes \widetilde{\nabla \theta}(y+h)) d y .
\end{aligned}
$$

By appealing to an obvious decomposition of $(\widetilde{\phi}(y+h) \otimes \widetilde{\nabla \theta}(y+h))-(\widetilde{\phi}(y) \otimes \widetilde{\nabla \theta}(y))$, it readily follows that

$$
|I| \leq c|h|\|\widetilde{\nabla \theta}\|_{C^{1}}\|\widetilde{\nabla u}\|_{(p-1) r}^{p-1}\left(\|\widetilde{\phi}\|_{r^{\prime}}+\|\nabla \widetilde{\phi}\|_{r^{\prime}}\right) .
$$

Hence

$$
\begin{array}{r}
v_{1}\left|\int\left(|\widetilde{\mathcal{D} u}(y)|^{p-2} \widetilde{\mathcal{D} u}(y)-|\widetilde{\mathcal{D} u}(y-h)|^{p-2} \widetilde{\mathcal{D} u}(y-h)\right):(\widetilde{\phi} \otimes \widetilde{\nabla \theta}) d y\right| \\
\leq C v_{1}|h|\|\nabla u\|_{(p-1) r}^{p-1}\|\nabla \widetilde{\phi}\|_{r^{\prime}} .
\end{array}
$$

From 8.23, 8.25 and 8.26 one deduces 8.20 . Simpler devices show that 8.21 holds. Alternatively, we may set $p=q=2$ in 8.20 .

Finally, by an obvious decomposition of the $\theta \phi$ terms, one shows that

$$
\left.\mid \int \tilde{f} \cdot(\widetilde{\theta \phi})(y+h)-(\widetilde{\theta \phi})(y)\right) d y|\leq C| h \mid\|f\|_{2}\|\nabla \widetilde{\phi}\|_{2}
$$

Alternatively, we could replace the right hand side of 88.27$)$ by $C|h|\|f\|_{p^{\prime}}\|\nabla \widetilde{\phi}\|_{p}$.

From 8.9, 8.21, 8.20) and 8.27) the estimate 8.6 follows. Theorem 8.2 is proved.

\section{Estimates for the "tangential derivatives" in terms of the data}

For convenience we set

$$
U_{0}(u, \pi)=\|\nabla u\|_{p}^{2}+\|\nabla u\|_{p}^{2(p-1)}+\|\pi\|_{p^{\prime}}^{2}+\|\pi\|_{p^{\prime}}^{p^{\prime}} .
$$

Below $\nabla_{*}$ denotes the gradient with respect to the variables $y_{j}, j=1,2$. Hence

$$
\begin{aligned}
& \left|\nabla_{*}(\widetilde{\nabla u})(y)\right|^{2}=\sum_{j=1,2} \sum_{i, k=1}^{3}\left(\partial_{j}(\widetilde{\nabla u})_{i k}\right)^{2}, \\
& \left|\nabla_{*}(\widetilde{\mathcal{D} u})(y)\right|^{2}=\sum_{j=1,2} \sum_{i, k=1}^{3}\left(\partial_{j}(\widetilde{\mathcal{D} u})_{i k}\right)^{2},
\end{aligned}
$$


and

$$
\left|\nabla_{*} \tilde{\pi}(y)\right|^{2}=\sum_{j=1,2}\left(\partial_{j} \tilde{\pi}\right)^{2} .
$$

In this section we prove the following result.

Theorem 9.1. Let $x_{0} \in \Gamma$ and let $a_{0}>0$ be as in Section 5 . Assume that $(u, \pi) \in$ $\mathbb{W}^{1, q}\left(Q_{a_{0}}\right) \times L^{r}\left(Q_{a_{0}}\right)$ is a weak solution of problem 2.1] in $Q_{a_{0}}$ which satisfies the boundary condition (1.4) in $\Gamma_{a_{0}}$. Then there is $a>0$ (which depends only on the $C^{2}$ norm of $\eta$ in the sphere $\left.\left\{x^{\prime}:\left|x^{\prime}\right|<a_{0}\right\}\right)$ such that

$$
\nabla_{*}(\widetilde{\nabla u}),|\widetilde{\mathcal{D} u}|^{p-2} \nabla_{*} \widetilde{\mathcal{D} u} \in L^{2}\left(Q_{a}\right) \quad \text { and } \quad \nabla_{*} \tilde{\pi} \in L^{r}\left(Q_{a}\right) .
$$

Moreover,

$$
\begin{gathered}
\left\|\nabla_{*}(\widetilde{\nabla u})\right\|_{2}^{2}+\left\||\widetilde{\mathcal{D} u}|^{(p-2) / 2} \nabla^{*} \widetilde{\mathcal{D} u}\right\|_{2}^{2} \leq C\left(\|\nabla u\|_{p}^{2}+\|\nabla u\|_{p}^{p}+\|\pi\|_{p^{\prime}}^{p^{\prime}}+\|f\|_{2}^{2}\right), \\
\left\|\nabla^{*} \widetilde{\pi}\right\|_{r}^{2} \leq C\left(1+\|\nabla u\|_{q}^{p-2}\right)\left(\|f\|_{2}^{2}+\|\nabla u\|_{p}^{2}+\|\nabla u\|_{p}^{p}+\|\pi\|_{p^{\prime}}^{p^{\prime}}\right) \\
+C\left(\|\nabla u\|_{(p-1) r}^{2(p-1)}+\|\pi\|_{r}^{2}\right),
\end{gathered}
$$

where the norms on the left hand sides concern the set $Q_{a / 2}$ and those on the right hand side concern $\Omega_{a}$.

\section{Remarks.}

- For convenience the reader may assume that the norms $\|\nabla u\|_{p},\|\pi\|_{p^{\prime}}$ and $\|f\|_{2}$ concern the whole of $\Omega$. On the other hand, it is worth noting that in the proofs below the norms $\|\nabla u\|_{(p-1) r}$ and $\|\pi\|_{r}$ in $\Omega_{a}$ are obtained via their equivalence with the norms $\|\widetilde{\nabla u}\|_{(p-1) r}$ and $\|\tilde{\pi}\|_{r}$ in $Q_{a}$ respectively. In fact, we may use these last quantities in the second inequality of (9.2).

- The constants $C$ depend on the $C^{2}$-norms of $\eta$ and $\theta$ in $Q_{a}$. However the $C^{2}$-norm of $\eta$ is bounded from above on $\Gamma$, hence is independent of the particular point $x_{0}$. On the other hand, the particular truncation function $\theta$ may be fixed once and for all in our proofs as a regular function equal to 1 for $\left|x^{\prime}\right| \leq a / 2$ and with compact support inside $I_{a}$. This shows that the dependence of the constants $C$ on $\theta$ is just a dependence on $a$.

- The smallness of $a$ in our proofs is required just to get a sufficiently small $\epsilon_{0}$ in (5.8). This value does not depend on the point $x_{0}$. Hence a strictly positive lower bound for $a$ exists, independently of $x_{0}$.

Proof of Theorem 9.1 From (7.30) and (8.5), and by setting in this last inequality $q=p$ (hence $r=p^{\prime}$ ), it readily follows that

$$
\begin{aligned}
\widetilde{A}_{0}^{2}+\widetilde{A}_{1}^{2} \leq & C|h|\left(\|\nabla u\|_{p}+\|\nabla u\|_{p}^{p / 2}\right)\left(\widetilde{A}_{0}+\widetilde{A}_{1}\right) \\
& +C h^{2}\left(\|\nabla u\|_{p}^{2}+\|\nabla u\|_{p}^{p}+\|\pi\|_{p^{\prime}}^{p^{\prime}}\right)+C h^{2}\|f\|_{2}^{2} .
\end{aligned}
$$

Hence

$$
\widetilde{A}_{0}^{2}+\widetilde{A}_{1}^{2} \leq C h^{2} U(u, \pi)+C h^{2}\|f\|_{2}^{2} .
$$


By $[8.5$, straightforward calculations show that

$$
\|(\tilde{\pi}(y)-\tilde{\pi}(y-h)) \tilde{\theta}\|_{r}^{2} \leq h^{2} \Lambda,
$$

where, for convenience, $\Lambda$ denotes the right hand side of the second inequality of 9.2 and the constants $C$ may be incorporated in $\Lambda$.

Let us write 9.3 in a more explicit form, by taking into account the definitions of $A_{0}$ and $A_{1}$. One has

$$
\begin{gathered}
\int_{Q_{a}}\left|\frac{\widetilde{\nabla u}(y)-\widetilde{\nabla u}(y-h)}{h^{2}}\right|^{2} \widetilde{\theta}^{2}(y) d y \\
\quad+\int_{Q_{a}}\left(|\widetilde{\mathcal{D} u}(y)|^{p-2}+|\widetilde{\mathcal{D} u}(y-h)|^{p-2}\right)\left|\frac{\widetilde{\mathcal{D} u}(y)-\widetilde{\mathcal{D} u}(y-h)}{h^{2}}\right|^{2} \widetilde{\theta}^{2}(y) d y \\
\leq C\left(\|\nabla u\|_{p}^{2}+\|\nabla u\|_{p}^{p}+\|\pi\|_{p^{\prime}}^{p^{\prime}}\right)+C\|f\|_{2}^{2} .
\end{gathered}
$$

Similarly, from $(9.4)$,

$$
\left[\int_{Q_{a}}\left|\frac{\tilde{\pi}(y)-\tilde{\pi}(y-h)}{h^{2}}\right|^{r} \widetilde{\theta}^{2}(y) d y\right]^{2 / r} \leq \Lambda .
$$

By fixing $\theta$ to be a regular function equal to 1 in $I_{a / 2}$ we get estimates like 9.5 and $(9.6)$ but with the following modifications of the left hand sides: We drop the function $\widetilde{\theta}$ and take integrals over $Q_{a / 2}$. This leads to the estimate of $\left\|D_{*}^{2} \widetilde{u}\right\|_{2}^{2}$ in 9.2 . In particular, this last result implies the convergence almost everywhere in $Q_{a / 2}$ of the differential quotients that appear on the left hand side of the modified equation 9.5 . By appealing to Fatou's lemma, we pass to the limit as $h$ goes to zero and obtain the estimate of the second term in the first inequality of 9.2). The estimate concerning $\pi$ follows from the modified 9.6 .

\section{Normal derivatives}

We set

$$
\left|D_{*}^{2} u(x)\right|^{2}=\left|\nabla_{*}(\nabla u(x))\right|^{2}+\left|\left(\partial_{3}^{2} u_{3}\right)(x)\right|^{2},
$$

where the derivatives are with respect to the $x$-variables, and also

$$
\xi(x)=\partial_{3}^{2} u(x), \quad \xi^{\prime}(x)=\left(\xi_{1}(x), \xi_{2}(x)\right), \quad M(x)=|\mathcal{D} u(x)| .
$$

Due to (5.8), we may replace (on the "right hand sides" of estimates) the derivatives $\partial_{k} \eta$ for $k=1,2$ simply by $\epsilon_{0}$. Recall that $\partial_{3} \eta=0$. In the same line, $c \epsilon_{0}$ and $\epsilon_{0}^{2}$ can be replaced by $\epsilon_{0}$.

We will use without specific mention the fact that

$$
\partial_{3} \widetilde{g}=\widetilde{\partial_{3} g} \text {. }
$$

Lemma 10.1. One has, a.e. in $Q_{a}$,

$$
\left|\widetilde{\xi}_{3}\right| \leq\left|\nabla_{*}(\widetilde{\nabla u})\right|+\epsilon_{0}\left|\tilde{\xi}^{\prime}\right| .
$$


Proof. From $\nabla \cdot u=0$ it follows that

$$
\widetilde{\xi}_{3}=-\partial_{3}\left(\widetilde{\partial_{1} u_{1}}+\widetilde{\partial_{2} u_{2}}\right) .
$$

On the other hand, from 5.9,

$$
\partial_{3}\left(\widetilde{\partial_{m} u_{l}}\right)=\partial_{m} \widetilde{\partial_{3} u_{l}}-\left(\partial_{m} \eta\right) \partial_{3}\left(\widetilde{\partial_{3} u_{l}}\right) .
$$

Hence, for $m, l \neq 3$,

$$
\left|\partial_{3} \widetilde{\partial_{m} u_{l}}\right| \leq\left|\nabla_{*}(\widetilde{\nabla u})\right|+\epsilon_{0}\left|\widetilde{\xi}^{\prime}\right| .
$$

By taking into account $[10.3$, the assertion follows.

Lemma 10.2. One has, a.e. in $Q_{a}$,

$$
\left|\widetilde{D_{*}^{2}} u(y)\right| \leq\left|\nabla_{*}(\widetilde{\nabla u})\right|+\epsilon_{0}\left|\widetilde{\xi^{\prime}}\right| .
$$

Proof. From [5.9,

$$
\mathcal{T}\left(\partial_{j} \partial_{k} u_{l}\right)=\partial_{k}\left(\widetilde{\partial_{j} u_{l}}\right)-\left(\partial_{k} \eta\right) \partial_{3}\left(\widetilde{\partial_{j} u_{l}}\right) .
$$

By appealing to the above estimates the conclusion follows easily. Note that if $j=k=$ $l=3$ the result follows from 10.2.

Straightforward calculations show that

$$
\partial_{k}\left(|\mathcal{D} u|^{p-2} \mathcal{D} u\right)=|\mathcal{D} u|^{p-2} \mathcal{D} \partial_{k} u+(p-2)|\mathcal{D} u|^{p-4}\left(\mathcal{D} u \cdot \mathcal{D} \partial_{k} u\right) \mathcal{D} u .
$$

By using (10.6), the $j$ th equation of 2.1] may be written in the form

$$
\begin{aligned}
& -v_{0} \sum_{k=1}^{3} \partial_{k}^{2} u_{j}-v_{1}|\mathcal{D} u|^{p-2} \sum_{k=1}^{3}\left(\partial_{k}^{2} u_{j}+\partial_{j k}^{2} u_{k}\right) \\
& \quad-(p-2) v_{1}|\mathcal{D} u|^{p-4} \sum_{l, m, k=1}^{3} \mathcal{D}_{l m} \mathcal{D}_{j k}\left(\partial_{m k}^{2} u_{l}+\partial_{l k}^{2} u_{m}\right)+\partial_{j} \pi=f_{j},
\end{aligned}
$$

where we have set $\mathcal{D}_{i j}=(\mathcal{D} u)_{i j}$. Let us write the first two equations 10.7) as follows:

$$
\begin{aligned}
v_{0} \partial_{3}^{2} u_{j}+v_{1}|\mathcal{D} u|^{p-2} \partial_{3}^{2} u_{j}+2(p-2) v_{1}|\mathcal{D} u|^{p-4} \mathcal{D}_{j 3} & \sum_{l=1}^{2} \mathcal{D}_{l 3} \partial_{3}^{2} u_{l} \\
& =F_{j}(x)+\partial_{j} \pi-f_{j},
\end{aligned}
$$

where the $F_{j}(x), j \neq 3$, are given by

$$
\begin{aligned}
F_{j}(x):= & -v_{0} \sum_{k=1}^{2} \partial_{k}^{2} u_{j}-v_{1}|\mathcal{D} u|^{p-2} \sum_{k=1}^{2} \partial_{k}^{2} u_{j}-v_{1}|\mathcal{D} u|^{p-2} \sum_{k=1}^{3} \partial_{j k}^{2} u_{k} \\
& -2(p-2) v_{1}|\mathcal{D} u|^{p-4}\left(\mathcal{D}_{33} \mathcal{D}_{j 3} \partial_{3}^{2} u_{3}+\sum_{\substack{l, m, k=1 \\
(m, k) \neq(3,3)}}^{3} \mathcal{D}_{l m} \mathcal{D}_{j k} \partial_{m k}^{2} u_{l}\right) .
\end{aligned}
$$


The measurable functions $F_{j}$ satisfy

$$
\left|F_{j}(x)\right| \leq c\left(v_{0}+v_{1}|M(x)|^{p-2}\right)\left|D_{*}^{2} u(x)\right|
$$

a.e. in $\Omega_{a}$. Hence, from 10.5 it follows that

$$
\left|\widetilde{F}_{j}\right| \leq\left(v_{0}+v_{1}|\tilde{M}|^{p-2}\right)\left(\left|\nabla_{*}(\widetilde{\nabla u})\right|+\epsilon_{0}\left|\tilde{\xi}^{\prime}\right|\right) .
$$

Lemma 10.3. One has, a.e. in $Q_{a}$,

$$
\left|\widetilde{\nabla_{*} \pi}\right| \leq\left|\nabla_{*} \tilde{\pi}\right|+\epsilon_{0}\left(v_{0}+v_{1}|\tilde{M}|^{p-2}\right)\left(\left|\nabla_{*}(\widetilde{\nabla u})\right|+\left|\widetilde{\xi^{\prime}}\right|\right)+\epsilon_{0}|\tilde{f}|
$$

and

$$
\left|\widetilde{\partial_{3} \pi}\right| \leq\left(v_{0}+v_{1}|\tilde{M}|^{p-2}\right)\left(\left|\nabla_{*}(\widetilde{\nabla u})\right|+\left|\widetilde{\xi^{\prime}}\right|\right)+|\widetilde{f}| .
$$

Proof. From 10.7 for $j=3$, we get an expression for $\partial_{3} \pi$. In particular, it follows that

$$
\begin{aligned}
\left|\partial_{3} \pi\right| \leq & c\left(v_{0}+(p-1) v_{1}|M(x)|^{p-2}\right)\left|D_{*}^{2} u(x)\right| \\
& +c(p-2) v_{1}|\mathcal{D} u(x)|^{p-2} \sum_{l=1}^{2}\left|\partial_{3}^{2} u_{l}\right|+\left|f_{3}(x)\right| .
\end{aligned}
$$

By transforming the inequality 10.13 from the $x$ to the $y$ variables and by appealing to 10.5 one gets 10.12. Since for $j=1,2$,

$$
\left|\widetilde{\partial_{j} \pi}\right| \leq\left|\partial_{j} \tilde{\pi}\right|+\epsilon_{0}\left|\widetilde{\partial_{3} \pi}\right|
$$

the estimate 10.11 holds.

Lemma 10.4. One has, a.e. in $Q_{a}$,

$$
\sum_{l=1}^{3}\left|\widetilde{\partial_{3}^{2} u_{l}}\right| \leq c\left|\nabla_{*}(\widetilde{\nabla u})\right|+\frac{c}{v_{0}+v_{1}|\tilde{M}|^{p-2}}\left(\left|\nabla_{*} \tilde{\pi}\right|+|\tilde{f}|\right) .
$$

Proof. Consider the system (10.8) in terms of the $y$ variables, i.e., the system

$$
v_{0} \widetilde{\xi}_{j}+v_{1}|\tilde{M}|^{p-2} \widetilde{\xi}_{j}+2(p-2) v_{1}|\tilde{M}|^{p-4} \widetilde{\mathcal{D}}_{j 3} \sum_{l=1}^{2} \widetilde{\mathcal{D}}_{l 3} \widetilde{\xi}_{l}=\widetilde{F}_{j}+\widetilde{\partial_{j} \pi}-\widetilde{f}_{j}
$$

We show that the $2 \times 2$ linear system 10.15 can be solved for the unknowns $\tilde{\xi}_{j}, j=1,2$, for almost all $y \in Q_{a / 2}$. The elements $\tilde{a}_{j l}$ of the matrix $\widetilde{A}$ of the system are given by

$$
\widetilde{a}_{j l}=\left(v_{0}+v_{1}|\tilde{M}|^{p-2}\right) \delta_{j l}+2(p-2) v_{1}|\tilde{M}|^{p-4} \widetilde{\mathcal{D}}_{l 3} \widetilde{\mathcal{D}}_{j 3}
$$

for $j, l \neq 3$. Note that $\tilde{a}_{j l}=\tilde{a}_{l j}$. One easily shows that

$$
\sum_{j, l=1}^{n-1} \tilde{a}_{j l} \lambda_{j} \lambda_{l}=\left(v_{0}+v_{1}|\tilde{M}|^{p-2}\right)|\lambda|^{2}+2(p-2) v_{1}|\tilde{M}|^{p-4}[(\widetilde{\mathcal{D} u}) \cdot \lambda]_{3}^{2} .
$$


Hence the matrix $\widetilde{A}$ is symmetric and positive definite. Moreover, the above identity shows that all the eigenvalues are larger than or equal to $\nu_{0}+v_{1}|\widetilde{M}|^{p-2}$. In particular,

$$
\operatorname{det} \tilde{A} \geq\left(v_{0}+v_{1}|\tilde{M}|^{p-2}\right)^{2} .
$$

Hence we deduce from (10.15), i.e. from

$$
\sum_{l=1}^{2} \widetilde{a}_{j l} \widetilde{\xi}_{l}=\widetilde{F}_{j}+\widetilde{\partial_{j} \pi}-\widetilde{f}_{j}
$$

that

$$
\sum_{l, j=1}^{2} \widetilde{a}_{j l} \widetilde{\xi}_{l} \widetilde{\xi}_{j}=\sum_{j=1}^{2}\left(\widetilde{F}_{j}+\widetilde{\partial_{j} \pi}-\widetilde{f}_{j}\right) \widetilde{\xi}_{j}
$$

Consequently,

$$
\left(v_{0}+v_{1}|\tilde{M}|^{p-2}\right)\left|\tilde{\xi}^{\prime}\right| \leq\left|\widetilde{F}_{j}+\widetilde{\partial_{j} \pi}-\widetilde{f}_{j}\right|
$$

a.e. in $Q_{a / 2}$. By appealing to 10.10 and 10.11 we show that

$$
\left(v_{0}+v_{1}|\tilde{M}|^{p-2}\right)\left|\tilde{\xi}^{\prime}\right| \leq c\left(v_{0}+v_{1}|\tilde{M}|^{p-2}\right)\left|\nabla_{*}(\widetilde{\nabla u})\right|+c\left|\nabla_{*} \tilde{\pi}\right|+c|\tilde{f}| .
$$

This estimate together with (10.2) proves 10.14).

Corollary 10.1. One has

$$
\begin{aligned}
\|\nabla u\|_{W^{1, r}\left(\Omega_{a / 2}\right)}^{2} \leq & C\left(1+\|\nabla u\|_{q}^{p-2}\right)\left(\|f\|_{2}^{2}+\|\nabla u\|_{p}^{2}+\|\nabla u\|_{p}^{p}+\|\pi\|_{p^{\prime}}^{p^{\prime}}\right) \\
& +C\left(\|\nabla u\|_{(p-1) r}^{2(p-1)}+\|\pi\|_{r}^{2}\right),
\end{aligned}
$$

where the norms on the right hand side concern $\Omega_{a}$.

Proof. From 10.14 and 9.2) (recall (10.1)) it follows that

$$
\|\nabla(\widetilde{\nabla u})\|_{L^{r}\left(Q_{a / 2}\right)}^{2} \leq C \Lambda,
$$

where $\Lambda$ is the right hand side of 9.2$)$. Clearly $\|\widetilde{\nabla u}\|_{W^{1, r}\left(Q_{a / 2}\right)}^{2}$ satisfies this last estimate. Hence (10.19) holds.

Next we appeal to the following algebraic lemma.

Lemma 10.5. Let $2<p<3$ and $p \leq q \leq 6$ and set

$$
\beta_{0}=\frac{3(p-2)}{6-p} \text {. }
$$

Define $\alpha$ by the equation

$$
\frac{1}{r}=\frac{1-\alpha}{p^{\prime}}+\frac{\alpha}{r^{*} /(p-1)}
$$

and set

$$
\beta=(p-1) \alpha .
$$

Then $\beta<\beta_{0}<1$ for each $q \geq p$.

The proof is left to the reader. 
Lemma 10.6. One has

$\|\pi\|_{r} \leq \epsilon^{-\frac{1}{1-\beta_{0}}}\|\pi\|_{p^{\prime}}^{\frac{1-\alpha}{1-\beta_{0}}}+c \epsilon^{\frac{1}{\beta_{0}}}\left(v_{0}\|\nabla u\|_{p}^{\frac{1}{p-1}}+v_{1}\|\mathcal{D} u\|_{r *}+\|\pi\|_{p^{\prime}}^{\frac{1}{p-1}}+\|f\|_{2}^{\frac{1}{p-1}}\right)$

for each $\epsilon>0$, where the norms may be taken in any open regular subset $\Omega_{0} \subset \Omega$.

Proof. From 10.21 it follows that

$$
\|\pi\|_{r} \leq \epsilon^{-1}\|\pi\|_{p^{\prime}}^{1-\alpha} \epsilon\|\pi\|_{\frac{r^{*}}{p-1}}^{\alpha} .
$$

By Young's inequality with exponents $1 /\left(1-\beta_{0}\right)$ and $1 / \beta_{0}$ we get

$$
\|\pi\|_{r} \leq \epsilon^{-\frac{1}{1-\beta_{0}}}\|\pi\|_{p^{\prime}}^{\frac{1-\alpha}{1-\beta_{0}}}+\epsilon^{\frac{1}{\beta_{0}}}\|\pi\|_{\frac{r^{*}}{p-1}}^{\frac{1}{p-1}} .
$$

Finally, by appealing to 4.7 with $s=r^{*}$ one proves 10.22). For convenience, we take into account that $r^{*} \leq 6$, hence $r^{*} /(p-1) \leq p$.

Lemma 10.7. Let $2 \leq p<3$ be fixed and define $r$ and $r^{*}$ as above, where $p \leq q \leq 6$. Set

$$
\alpha_{0}=1-\frac{2}{15}(3-p), \quad \gamma_{0}=\frac{1+\alpha_{0}}{2} .
$$

Then, for an arbitrary $g$,

$$
\|g\|_{(p-1) r}^{p-1} \leq\left(\epsilon^{-1}\left(1+\|g\|_{p}^{p-1}\right)^{\left(\gamma_{0} / \alpha_{0}\right)^{\prime}}+(2 \epsilon)^{\gamma_{0} / \alpha_{0}}\left(1+\|g\|_{r^{*}}^{\gamma_{0}}\right) .\right.
$$

Proof. Define $\alpha$ by

$$
\frac{1}{(p-1) r}=\frac{1-\alpha}{p}+\frac{\alpha}{r^{*}} .
$$

Then

$$
(p-1) \alpha=1-\frac{2\left(\frac{1}{p}-\frac{1}{3}\right)}{\frac{1}{p}-\left(\frac{p-2}{2 q}+\frac{1}{6}\right)} .
$$

The maximum of the above quantity is attained for $q=6$ (we may get better exponents by assuming that $\left.q \leq q_{\infty}\right)$. By setting $q=6$ one easily shows that $(p-1) \alpha \leq \alpha_{0}$.

From the definition of $\alpha$ and interpolation it follows that

$$
\|g\|_{(p-1) r}^{p-1} \leq\|g\|_{p}^{(p-1)(1-\alpha)}\|g\|_{r^{*}}^{(p-1) \alpha} .
$$

In particular,

$$
\|g\|_{(p-1) r}^{p-1} \leq \epsilon^{-1}\left(1+\|g\|_{p}^{p-1}\right) \cdot \epsilon\left(1+\|g\|_{r^{*}}^{\alpha_{0}}\right) .
$$

The conclusion follows by appealing to Young's inequality with exponents $\left(\gamma_{0} / \beta_{0}\right)^{\prime}$ and $\gamma_{0} / \beta_{0}$. 
For convenience we denote in the following by

$$
P=P\left(\|\nabla u\|_{p},\|\pi\|_{p^{\prime}}\right)
$$

very simple expressions (that can be reduced, for instance, to low order polynomials) that depend only on the two quantities indicated above. Explicit expressions follow immediately from our calculations.

From 10.22 we get, with an obvious simplified notation,

$$
\|\pi\|_{r} \leq C(\epsilon)(P+\|f\|)+c \epsilon^{1 / \beta_{0}}\|\nabla u\|_{r^{*}},
$$

where the norms of $\pi$ and $\nabla u$ may concern any $\Omega_{0}$ as above.

By (10.26) with $g=\nabla u$, and by (10.27), we obtain the following estimate:

Lemma 10.8. One has

$$
\|\nabla u\|_{(p-1) r}^{p-1}+\|\pi\|_{r} \leq C(\epsilon)\left(P+\|f\|_{2}\right)+c\left(\epsilon^{\gamma_{0} / \alpha_{0}}+\epsilon^{1 / \beta_{0}}\right)\left(1+\|\nabla u\|_{r^{*}}\right),
$$

where the norms could be taken in any $\Omega_{0}$ as above.

By taking into account the continuous immersion $W^{1, r} \subset L^{r}$, and by appealing to 10.19 and (10.27), we get the following result:

Proposition 10.1. One has

$$
\begin{aligned}
\|\nabla u\|_{W^{1, r}\left(\Omega_{a / 2}\right)}+\|\pi\|_{L^{r}\left(\Omega_{(a) / 2}\right) \leq} & C(\epsilon)\left(1+\|\nabla u\|_{q}^{(p-2) / 2}\right)\left(P+\|f\|_{2}\right) \\
& +c\left(\epsilon^{\gamma_{0} / \alpha_{0}}+\epsilon^{1 / \beta_{0}}\right)\left(1+\|\nabla u\|_{W^{1, r}\left(\Omega_{a}\right)}\right) .
\end{aligned}
$$

In particular

$$
\begin{aligned}
\|u\|_{W^{2, r}(\Omega)}+\|\pi\|_{L^{r}(\Omega)} \leq & N\left[C(\epsilon)\left(1+\|\nabla u\|_{q}^{(p-2) / 2}\right)\left(P+\|f\|_{2}\right)\right. \\
& \left.+c\left(\epsilon^{\gamma_{0} / \alpha_{0}}+\epsilon^{1 / \beta_{0}}\right)\left(1+\|\nabla u\|_{W^{1, r}(\Omega)}\right)\right],
\end{aligned}
$$

where $N$ is the number of sets of type $\Omega_{a / 2}$ plus the number of spheres contained in the interior of $\Omega$ sufficient to cover $\Omega$. It is worth noting that $W^{2,2}$ interior regularity for $u$ is trivial. Finally, by fixing a sufficiently small value of $\epsilon$ we prove that 2.5 holds.

Note that by the second inequality of $(9.2)$ and $(10.28)$ it readily follows that

$$
\left\|\nabla^{*} \tilde{\pi}\right\|_{L^{r}\left(Q_{a / 2}\right)} \leq C\left(1+\|\nabla u\|_{q}^{(p-2) / 2}\right)\left(P+\|f\|_{2}\right) .
$$

Finally, from 10.12 and 10.18 one gets

$$
\left|\widetilde{\partial_{3} \pi}\right| \leq c\left(v_{0}+v_{1}|\tilde{M}|^{p-2}\right)\left|\nabla_{*}(\widetilde{\nabla u})\right|+c\left|\nabla_{*} \tilde{\pi}\right|+c|\widetilde{f}|
$$

a.e. in $Q_{a}$. Set

$$
\bar{p}=\frac{2 q}{2(p-2)+q}
$$


From Hölder's inequality

$$
\left.\left\||\tilde{M}|^{p-2}\left(\nabla_{*}(\widetilde{\nabla u})\right)\right\|_{\bar{p}} \leq\|\tilde{M}\|_{q}^{p-2} \| \nabla_{*}(\widetilde{\nabla u})\right) \|_{2} .
$$

It readily follows that

$$
\left.\left\|\widetilde{\partial_{3} \pi}\right\|_{\bar{p}} \leq c\left(1+\|\tilde{M}\|_{q}^{p-2}\right) \| \nabla_{*}(\widetilde{\nabla u})\right)\left\|_{2}+c\right\| \nabla_{*} \tilde{\pi}\left\|_{\bar{p}}+c\right\| \tilde{f} \|_{2},
$$

where all the norms are taken in $Q_{a / 2}$. From 10.34 it follows that $\|\nabla \tilde{\pi}\|_{L^{\bar{p}}\left(Q_{a / 2}\right)}$ is bounded by the right hand side of (10.34) (replace $c$ by $c+1$ and recall $(10.1)$ ). By appealing to the first estimate in 9.2 ) one shows that the right hand side of [10.34) is bounded by the right hand side of 10.35 below. Hence

$$
\|\nabla \pi\|_{L^{\bar{p}}\left(\Omega_{a / 2}\right)} \leq C\left(1+\|\nabla u\|_{q}^{p-2}\right)\left(P+\|f\|_{2}\right)+\left\|\nabla_{*} \tilde{\pi}\right\|_{\bar{p}},
$$

where a simple expression for the term $P=P\left(\|\pi\|_{p^{\prime}},\|\nabla u\|_{p}\right)$ is easily obtained.

By appealing to 10.31 and by taking into account the interior regularity of the weak solutions one proves that (2.6) holds. Thus Theorem 2.1 is completely proved. Moreover, Theorem 2.2 follows by setting $q=p$ in Theorem 2.1

Remark. It is quite easy to show that $\nabla \pi \in L_{\text {loc }}^{p_{1}}(\Omega)$ where $p_{1}=12 /(p+4)$. Note that $p_{1}>\bar{p}$ (actually stronger results hold).

\section{Proof of Theorem 2.3}

Since we know that weak solutions belong to $W^{1, p}$, the above results are effective if we set $q=p$. In this particular case $r=p^{\prime}$ and by 10.19 and a Sobolev immersion theorem one gets $u \in W^{1,\left(p^{\prime}\right)^{*}}$. If $0<p<3$ it follows that $\left(p^{\prime}\right)^{*}>p$. hence the above results also hold for $q=\left(p^{\prime}\right)^{*}$. This same argument, used again and again, leads to a bootstrap argument.

We advise the reader that (as usual in the framework of Sobolev embedding theorems and similar theories) it is preferable to work with the inverse of the integrability exponents, rather than with the exponents themselves. Below, \|\|$_{k, s}$ denotes the norm in the Sobolev space $W^{k, s}(\Omega)$.

We define $r=r(q)$ by (8.4), and the Sobolev embedding exponent $r^{*}$ by (8.3). Hence $r^{*}=r^{*}(q)$ is defined by

$$
r^{*}(q)=\frac{6 q}{3(p-2)+q}
$$

for $p \leq q \leq 6$. In the following $r=r(q)$ and $r^{*}=r^{*}(q)$.

Note that $r^{*}(p) \geq p$ for $p \leq 3\left(r^{*}(3)=3\right)$, and $q \mapsto r^{*}(q)$ is a strictly increasing function for each fixed $p>2$. In particular, for $p<3$,

$$
r^{*}(q)>p \quad \text { if } q>p \text { and } p \leq 3 .
$$

Theorem 2.1 shows that if $u \in W^{1, q}$ then $u \in W^{2, r}$. Moreover, by [2.5],

$$
\|u\|_{2, r} \leq C\left(1+\|\nabla u\|_{q}^{(p-2) / 2}\right)\left(P+\|f\|_{2}\right) .
$$


Hence, by a Sobolev embedding theorem, $u \in W^{1, r^{*}}$ and

$$
\|u\|_{1, r^{*}} \leq c_{0}\|u\|_{2, r} \leq C\left(1+\|\nabla u\|_{q}^{(p-2) / 2}\right)\left(P+\|f\|_{2}\right) .
$$

Since $1+2 /(p-2) \leq r \leq 2$, the distinct values of the embedding constants $c_{0}$ are bounded from above by a constant independent of $r$.

This shows the following result.

Lemma 11.1. If a solution $u$ belongs to $W^{1, q}$ then it belongs to $W^{1, r^{*}}$, where $r^{*}(q)$ is given by 11.1, and moreover

$$
\|u\|_{1, r^{*}} \leq C\left(1+\|\nabla u\|_{q}^{(p-2) / 2}\right)\left(P+\|f\|_{2}\right) .
$$

Since $p \geq 2$ the function $r^{*}(q)$ is increasing and bounded from above (for instance, by 6). Next we define the increasing sequence

$$
q_{1}=p, \quad q_{n+1}=r^{*}\left(q_{n}\right) .
$$

Clearly

$$
q_{\infty}=3(4-p)
$$

is a fixed point of $r^{*}, r^{*}\left(q_{\infty}\right)=q_{\infty}$, and moreover

$$
\lim _{n \rightarrow \infty} q_{n}=q_{\infty}
$$

From 11.2 it follows that

$$
\|u\|_{1, q_{n+1}} \leq c\left(1+\|u\|_{1, q_{n}}^{(p-2) / 2}\right)(P+\|f\|) .
$$

Next we appeal to an induction argument. Note that for $n=1$ one has

$$
\|u\|_{1, q_{1}}=\|u\|_{1, p}
$$

If we are able to show that the quantities $a_{n}=\|u\|_{1, q_{n}}$, at least for large values of $n$, are uniformly bounded by a finite number $L$, then well known results from real analysis, together with (11.5), yield

$$
\|u\|_{1, q_{\infty}} \leq L .
$$

For convenience we write 11.6 in the form

$$
\|u\|_{1, q_{n+1}} \leq b+b\|u\|_{1, q_{n}}^{\alpha}
$$

where $b=c(P+\|f\|)$ and

$$
\alpha=\frac{p-2}{2} .
$$

Note that $0 \leq \alpha<1$ provided that $2 \leq p<4$. Denote by $\lambda$ the (unique) solution of the equation $\lambda=b+b \lambda^{\alpha}$. By (11.6) one has $a_{n+1} \leq b+b a_{n}^{\alpha}$. Set $b_{1}=a_{1}$ and $b_{n+1}=b+b b_{n}^{\alpha}$. Clearly $a_{n} \leq b_{n}$ for each $n$. It is easily seen that if $b_{1}<\lambda$ then the sequence $b_{n}$ is strictly increasing and converges to the fixed point $\lambda$. If $b_{1}>\lambda$ then the 
sequence decreases to the value $\lambda$. Hence the sequence $b_{n}$ converges to $\lambda$, so $a_{n}<2 \lambda$ for large values of $n$. On the other hand, one easily shows that

$$
\lambda \leq 2 b+(2 b)^{1 /(1-\alpha)} .
$$

Hence, under the hypothesis of Theorem 2.3 , one has

$$
\|u\|_{1, q_{\infty}} \leq c(P+\|f\|)+c(P+\|f\|)^{2 /(4-p)} .
$$

Theorem 2.3 now follows by applying once more Theorem 2.1, this time with $q=q_{\infty}$ given by (11.4). In this case the equation (8.4) shows that $r=r\left(q_{\infty}\right)=l$, with $l$ given by (2.12). Hence, from 2.5, it follows that

$$
\|u\|_{W^{2, r}(\Omega)} \leq P\left(1+\|f\|^{2 /(4-p)}\right) .
$$

Finally, from (2.6) written with $q=q_{\infty}$, together with (11.9), one obtains 2.13).

\section{Proof of Theorem 2.4}

We show here the a priori estimate that leads to the desired results. A complete proof follows by using standard devices.

Since

$$
\int_{\Omega}(u \cdot \nabla) u \cdot u d x=0,
$$

it readily follows that all the estimates for weak solutions, stated in Section 2, hold here.

On the other hand, by Hölder's inequality,

$$
\|(u \cdot \nabla) u\| \leq\|u\|_{p^{*}}\|\nabla u\|_{s}
$$

where $s=6 p /(5 p-6)$. By well known embedding theorems it follows that

$$
\|(u \cdot \nabla) u\| \leq\|u\|_{W^{1, p}\|u\|_{W^{3 / 2, p^{\prime}}} .}
$$

By appealing, in particular, to the compact embedding of $W^{2, p^{\prime}}$ into $W^{3 / 2, p^{\prime}}$ one proves that to each positive real $\epsilon$ there corresponds a positive $C_{\epsilon}$ such that

$$
\|(u \cdot \nabla) u\|_{2} \leq\|u\|_{W^{1, p}}\left(C_{\epsilon}\|u\|_{W^{1, p}}+\epsilon\|u\|_{W^{2, p^{\prime}}}\right) .
$$

Next we treat the term $(u \cdot \nabla) u$ as a "right hand side", by adding it to the external forces $f$ in the estimate 2.9. This gives

$$
\|u\|_{2, p^{\prime}} \leq P(1+\|f\|)+C_{\epsilon} P+\epsilon C_{0}\left(1+\|u\|_{1, p}^{p / 2}\right)\|u\|_{2, p^{\prime}},
$$

where the quantities $P=P\left(\|\nabla u\|_{p},\|\pi\|_{p^{\prime}}\right)$ may change from equation to equation and $C_{0}$ denotes a particular constant $C$. By fixing a sufficiently small value of $\epsilon$ we easily show that

$$
\|u\|_{2, p^{\prime}} \leq P(1+\|f\|)
$$

for some $P$. From $(12.1)$ it follows that

$$
\|(u \cdot \nabla) u\| \leq P(1+\|f\|) .
$$

Consequently, if in the estimates stated in Theorems 2.12 .3 we replace $f$ by $f+(u \cdot \nabla) u$ this simply leads to replacing $\|f\|$ by $P\|f\|$. 


\section{The evolution Navier-Stokes equation}

It is well known, and easily shown, that under suitable conditions one has $\partial_{t} u \in$ $L^{2}\left(0, T ; L^{2}(\Omega)\right)$. See, for instance, Theorem 7.2 in [18] (for the reader's convenience, we give a straightforward proof below). Since our regularity results for the second order derivatives $\left|\nabla^{2} u\right|$ of solutions $u$ to the stationary problem 2.1 hold if $f \in L^{2}(\Omega)$, corresponding regularity results for solutions to the evolution problem follow immediately by considering $\partial_{t} u$ as a "right hand side".

In what follows we merely prove the a priori estimates that lead to Theorems 2.5 and 2.6. Complete proofs are done by applying the estimates to the approximate solutions obtained by the Faedo-Galerkin method. By now this is a well known device. See, for instance, [22] and [27, Section 2].

Multiplication by $u$ and integration in $\Omega$ followed by suitable integrations by parts show that

$$
\frac{1}{2} \frac{d}{d t}\|u(t)\|^{2}+\frac{\nu_{0}}{2}\|\mathcal{D} u\|^{2}+\frac{\nu_{1}}{2}\|\mathcal{D} u\|_{p}^{p}=\int_{\Omega} f u d x .
$$

By integration of 13.1 with respect to time, one gets the following result:

Lemma 13.1. Let $u$ be a weak solution to problem 2.16) under the boundary condition (1.4) plus $x^{\prime}$-periodicity. Then $u$ satisfies the estimate

$$
\begin{aligned}
\|u(t)\|_{L^{\infty}\left(0, T ; L^{2}\right)}^{2}+v_{0}\|u\|_{L^{2}\left(0, T ; H^{1}\right)}^{2} & +v_{1}\|u\|_{L^{p}\left(0, T ; W^{1, p}\right)}^{p} \\
& \leq c\left(\|u(0)\|^{2}+\frac{1}{v_{0}}\|f\|_{L^{2}\left(0, T ; H^{-1}\right)}^{2}\right) .
\end{aligned}
$$

Next we prove a stronger estimate "in time" (see (13.4). A complete proof of this estimate is done by passing through the solutions of a suitable family of approximate problems. This can be done by appealing to a Faedo-Galerkin procedure as, for instance, in Theorem 2.2 of [27].

We define $\mathcal{M}$ by the equation

$$
\begin{aligned}
\mathcal{M}^{2}= & 2 \exp \left\{\frac{c}{v_{1}} \int_{0}^{T}\|\mathcal{D} u\|_{p}^{4-p} d t\right\} \\
& \cdot\left\{v_{0}\left\|\mathcal{D} u_{0}\right\|^{2}+v_{1}\left\|\mathcal{D} u_{0}\right\|_{p}^{p}+c \int_{0}^{T}\|f(t)\|^{2} d t\right\} .
\end{aligned}
$$

Note that, by (13.2), the first integral on the right hand side of 13.3 can be estimated in terms of the data since $4-p \leq p$.

One has the following result:

Lemma 13.2. Let $u$ be as in Lemma 13.1 and assume that $u_{0} \in V_{p}$, 2.17 holds and $f \in L^{2}\left(0, T ; L^{2}\right)$. Then

$$
\left\|\partial_{t} u\right\|_{L^{2}\left(0, T ; L^{2}\right)}^{2}+v_{0}\|\nabla u\|_{L^{\infty}\left(0, T ; L^{2}\right)}^{2}+v_{1}\|\nabla u\|_{L^{\infty}\left(0, T ; L^{p}\right)}^{p} \leq c \mathcal{M}^{2}
$$

Proof. By suitable integrations by parts, it follows that

$-\int_{\Omega}\left[\nabla \cdot\left(v_{0} \nabla u+v_{1}|\mathcal{D} u|^{p-2} \mathcal{D} u\right)+\nabla \pi\right] \cdot \frac{\partial u}{\partial t} d x=\frac{\nu_{0}}{2} \frac{d}{d t}\|\mathcal{D} u\|^{2}+\frac{v_{1}}{2 p} \frac{d}{d t}\|\mathcal{D} u\|_{p}^{p}$. 
On the other hand,

$$
\int_{\Omega}|(u \cdot \nabla) u|^{2} d x \leq c\|u\|_{2 p /(p-2)}^{2}\|\nabla u\|_{p}^{2}
$$

Furthermore,

$$
\|u\|_{2 p /(p-2)} \leq c\|u\|_{p^{*}}
$$

provided that 2.17 holds.

Remark. The assumption 2.17) is superfluous if we drop the term $(u \cdot \nabla) u$ from equation 1.1.

By appealing to a Sobolev embedding theorem together with 4.1), one shows that

$$
\|(u \cdot \nabla) u\| \leq c\|\mathcal{D} u\|_{p}^{2} .
$$

Hence, from 2.16 and 13.5, one gets

$$
\left\|\partial_{t} u\right\|^{2}+v_{0} \frac{d}{d t}\|\nabla u\|^{2}+v_{1} \frac{d}{d t}\|\mathcal{D} u\|_{p}^{p} \leq c\left(\|f\|^{2}+\|\mathcal{D} u\|_{p}^{4-p}\|\mathcal{D} u\|_{p}^{p}\right) .
$$

From 13.9 straightforward, well known manipulations show that

$$
\left\|\partial_{t} u\right\|_{L^{2}\left(0, T ; L^{2}\right)}^{2}+v_{0}\|\mathcal{D} u\|_{L^{\infty}\left(0, T ; L^{2}\right)}^{2}+v_{1}\|\mathcal{D} u\|_{L^{\infty}\left(0, T ; L^{p}\right)}^{p} \leq \mathcal{M}^{2} .
$$

Finally, by (4.1), 13.4) follows for some constants $c$.

In particular, the following result holds.

Corollary 13.1. The constants of type $P$ (see (2.2)) are now time depending uniformly bounded functions $P=P(t)$ in $(0, T)$.

Proof of Theorem 2.5. One has, almost everywhere in ]0, $T$,

$$
-v_{0} \Delta u-v_{1} \nabla \cdot\left(|\mathcal{D} u|^{p-2} \mathcal{D} u\right)+\nabla \pi=f(x)-(u \cdot \nabla) u-\partial_{t} u .
$$

Hence, by taking into account $(2.9)$, one shows that for each $t \in] 0, T[$,

$$
\|u\|_{2, p^{\prime}} \leq P(t)\left(1+\|f\|+\|(u \cdot \nabla) u\|+\left\|\partial_{t} u\right\|\right) .
$$

Consequently, by (13.4) and (13.8), straightforward calculations show that the norm $\|u\|_{L^{2}\left(0, T ; W^{\left.2, p^{\prime}\right)}\right.}$ is bounded.

Similarly, by appealing to 2.10, one proves that $\|\nabla \pi\|_{L^{2}\left(0, T ; L^{\left.p_{0}\right)}\right.}$ is bounded.

Proof of Theorem 2.6. Now $p^{\prime}$ is replaced by $l$ and we combine (13.4) with (2.11) to get

$$
\|u\|_{2, l} \leq P(t)\left(1+\|f\|^{2 /(4-p)}+\|\mathcal{D} u\|^{4 /(4-p)}+\left\|\partial_{t} u\right\|^{2 /(4-p)}\right),
$$

a.e. in ]0, $T$ [. Hence, by taking the $(4-p)$ th power of both sides of $(13.12)$ and by integrating in $\Omega$, one shows that $\|u\|_{L^{4-p}\left(0, T ; W^{2, l}\right)}$ is bounded. By appealing to 2.13) one proves that $\|\nabla \pi\|_{L^{2(4-p) / p\left(0, T ; L^{m}\right)}}$ is bounded. 


\section{Appendix 1: The Stokes principle}

Let us briefly illustrate this principle, in a postulational form, by borrowing from Serrin's fundamental work [31, p. 231].

1. $T$ is a continuous function of the deformation tensor $\mathcal{D}$, and is independent of all other kinematic quantities.

2. $T$ does not depend explicitly on the position $x$ (spatial homogeneity).

3 . There is no preferred direction in space (isotropy).

4. When $\mathcal{D}=0, T$ reduces to $-\pi I$.

For further comments and references, see Serrin's work above.

\section{Appendix 2}

This appendix is connected with the proof of Theorem 8.1 to which the reader is referred. We recall that Nečas' result (see [25]) states that if $g \in W^{-1, r}(Q)$ and $\nabla g \in W^{-1, r}(Q)$ then $g \in L^{r}(Q)$, and moreover

$$
\|g\|_{r} \leq c\left(\|g\|_{-1, r}+\|\nabla g\|_{-1, r}\right) .
$$

We claim that if $g$ is a distribution in $Q$ and $\nabla g \in W^{-1, r}(Q)$ then $g \in L^{r}(Q)$, and moreover

$$
\|g\|_{L_{\#}^{r}} \leq\|\nabla g\|_{-1, r} .
$$

However, we do not take this short cut. Actually, we show that $(\tilde{\pi}(y)-\tilde{\pi}(y-h)) \tilde{\theta} \in$ $W^{-1, r}\left(Q_{a}\right)$, and that the corresponding norm is bounded by the right hand side of 8.8 . We apply $(15.1)$ with $g=(\widetilde{\pi}(y)-\tilde{\pi}(y-h)) \widetilde{\theta}$ to prove (8.8).

Straightforward computations show that

$$
\begin{aligned}
\mid \int(\tilde{\pi}(y)- & \tilde{\pi}(y-h)) \tilde{\theta} \cdot \tilde{\phi} d y \mid \\
& \leq\|\widetilde{\theta}\|_{C_{0}}\|\tilde{\pi}\|_{p^{\prime}}\|\widetilde{\phi}(y+h)-\widetilde{\phi}(y)\|_{p}+|h|\|\nabla \widetilde{\theta}\|_{C_{0}}\|\tilde{\pi}\|_{p^{\prime}}\|\widetilde{\phi}\|_{p} \\
& \leq|h|\|\tilde{\theta}\|_{C_{1}}\|\tilde{\pi}\|_{p^{\prime}}\left(\|\tilde{\phi}\|_{p}+\|\nabla \widetilde{\phi}\|_{p}\right)
\end{aligned}
$$

for each scalar field $\widetilde{\phi} \in C_{0}^{2}\left(Q_{a}\right)$. Hence,

$$
\|(\tilde{\pi}(y)-\tilde{\pi}(y-h)) \widetilde{\theta}\|_{-1, p^{\prime}} \leq|h|\|\widetilde{\theta}\|_{C_{1}}\|\tilde{\pi}\|_{p^{\prime}} .
$$

Since $p^{\prime} \leq r$, it follows from 8.7 that $\|\nabla[(\tilde{\pi}(y)-\tilde{\pi}(y-h)) \tilde{\theta}]\|_{-1, p^{\prime}}$ is bounded by the right hand side of 8.77. Consequently, by the above result of Nečas (applied with exponent $\left.p^{\prime}\right)$ it follows that $\|(\tilde{\pi}(y)-\tilde{\pi}(y-h)) \widetilde{\theta}\|_{p^{\prime}}$ is bounded by a constant times the right hand side of 8.7 . Since $L^{p^{\prime}} \subset W^{-1, r}$, one shows that $\|(\tilde{\pi}(y)-\tilde{\pi}(y-h)) \tilde{\theta}\|_{-1, r}$ is bounded by a constant times the right hand side of 8.7 . 
Added in proof. For further developments of the results proved here we refer to the forthcoming paper [7].

Acknowledgments. These results were obtained during a stay, in February-July 2006, at the "Centro de Matemática e Aplicações Fundamentais", Lisbon Faculty of Sciences, under the award of a "Gulbenkian Professorship" from the Fundação Calouste Gulbenkian. The author is grateful to both the institutions for their kind hospitality and support during the preparation of this work.

We also express our thanks to the referees for their remarks and suggestions. In fact, since their comments constitute a very good introduction to our work, we took the liberty of incorporating them in our paper.

\section{References}

[1] Acerbi, E., Mingione, G.: Regularity results for stationary electrorheological fluids. Arch. Ration. Mech. Anal. 164, 213-259 (2002) Zbl 1038.76058 MR 1930392

[2] Beirão da Veiga, H.: Regularity for Stokes and generalized Stokes systems under nonhomogeneous slip type boundary conditions. Adv. Differential Equations 9, 1079-1114 (2004) Zbl 1103.35084 MR 2098066

[3] Beirão da Veiga, H.: On the regularity of flows with Ladyzhenskaya shear dependent viscosity and slip and non-slip boundary conditions. Comm. Pure Appl. Math. 58, 552-577 (2005) Zbl 1075.35045 MR 2119869

[4] Beirão da Veiga, H.: Navier-Stokes equations with shear thickening viscosity. Regularity up to the boundary. J. Math. Fluid Mech., in press, DOI 10.1007/s00021-008-0257-2

[5] Beirão da Veiga, H.: Navier-Stokes equations with shear thinning viscosity. Regularity up to the boundary. J. Math. Fluid Mech., in press, DOI 10.1007/s00021-008-0258-1

[6] Beirão da Veiga, H.: Turbulence models, $p$-fluid flows, and $W^{2, l}$ regularity of solutions. Comm. Pure Appl. Anal. 8, 769-783 (2009)

[7] Beirão da Veiga, H., Kaplický, P., Růžička, M.: Boundary regularity of shear thickening flow. Preprint, Charles Univ. Prague, MATH-kma-2008/277 340.8

[8] Consiglieri, L.: Weak solutions for a class of non-Newtonian fluids with energy transfer. J. Math. Fluid Mech. 2, 267-293 (2000) Zbl 0974.35090 MR 1781916

[9] Consiglieri, L.: Existence for a class of non-Newtonian fluids with a nonlocal friction boundary condition. Acta Math. Sinica 22, 523-534 (2006) Zbl 1106.35012 MR 2214374

[10] Consiglieri, L.: Steady-state flows of thermal viscous incompressible fluids with convectiveradiation effects. Math. Models Methods Appl. Sci. 16, 2013-2027 (2006) Zbl 1112.35038 MR 2287338

[11] Crispo, F.: On the regularity of shear thickening viscous flows. Chin. Ann. of Math. Ser. B, in press

[12] Fuchs, M., Seregin, G.: Variational Methods for Problems from Plasticity Theory and for Generalized Newtonian Fluids. Lecture Notes in Math. 1749, Springer, Berlin (2000) Zbl 0964.76003 MR 1810507

[13] Hughes, T. J. R., Mazzei, L., Oberai, A. A.: The multiscale formulation of large eddy simulation: Decay of homogeneous isotropic turbulence. Phys. Fluids 13, 505-512 (2001)

[14] Ladyzhenskaya, O. A.: On nonlinear problems of continuum mechanics. In: Proc. Int. Congr. Math. (Moscow, 1966), Mir, Moscow, 560-573 (1968) (in Russian); English transl., Amer. Math. Soc. Transl. (2) 70, 73-89 (1968) Zbl 0194.41701 MR 0239291

[15] Ladyzhenskaya, O. A.: Sur de nouvelles équations dans la dynamique des fluides visqueux et leur résolution globale. Trudy Mat. Inst. Steklov. 102, 85-104 (1967) (in Russian) Zbl 0202.37802 MR 0226907 
[16] Ladyzhenskaya, O. A.: Sur des modifications des équations de Navier-Stokes pour des grands gradients de vitesses. Zap. Nauchn. Sem. LOMI 7, 126-154 (1968) (in Russian) Zbl 0195.10602 MR 0241832

[17] Ladyzhenskaya, O. A.: The Mathematical Theory of Viscous Incompressible Flow. 2nd ed., Gordon and Breach, New York (1969) Zbl 0184.52603 MR 0254401

[18] Ladyzhenskaya, O. A.: Some results on modifications of three-dimensional Navier-Stokes equations. In: Nonlinear Analysis and Continuum Mechanics, G. Buttazzo et al. (eds.), Springer, New York, 73-84 (1998) MR 1600944

[19] Ladyzhenskaya, O. A., Seregin, G. A.: On regularity of solutions to two-dimensional equations of the dynamics of fluids with nonlinear viscosity. Zap. Nauchn. Sem. POMI 259, 145 166 (1999) (in Russian) Zbl 1060.76005 MR 1754361

[20] Lions, J.-L.: Sur certaines équations paraboliques non linéaires. Bull. Soc. Math. France 93, 155-175 (1965) Zbl 0132.10601 MR 0194760

[21] Lions, J.-L.: Quelques Méthodes de Résolution des Problèmes aux Limites Non Linéaires. Dunod, Paris (1969) Zbl 0189.40603 MR 0259693

[22] Málek, J., Nečas, J., Rokyta, M., Růžička, M.: Weak and Measure-Valued Solutions to Evolutionary PDEs, Appl. Math. Math. Comput. 13, Chapman and Hall, London (1996) Zbl 0851.35002 MR 1409366

[23] Málek, J., Nečas, J., Růžička, M.: On weak solutions to a class of non-Newtonian incompressilble fluids in bounded three-dimensional domains: the case $p \geq 2$. Adv. Differential Equations 6, 257-302 (2001) Zbl 1021.35085 MR 1799487

[24] Málek, J., Růžička, M., Shelukhin, V. V.: Herschel-Bulkley fluids: Existence and regularity of steady flows. Math. Models Methods Appl. Sci. 15, 1845-1861 (2005) Zbl 1098.76008 MR 2189915

[25] Nečas, J.: Équations aux Dérivées Partielles. Univ. Montréal, Montréal (1965) Zbl 0147.07801

[26] Nirenberg, L.: On elliptic partial differential equations. Ann. Scuola Norm. Sup. Pisa 13, 116162 (1959) Zbl 0088.07601 MR 0109940

[27] Parés, C.: Existence, uniqueness and regularity of solutions of the equations of a turbulence model for incompressible fluids. Appl. Anal. 43, 245-296 (1992) Zbl 0739.35075 MR 1284321

[28] Rajagopal, K. R.: Mechanics of non-Newtonian fluids. In: Recent Developments in Theoretical Fluid Mechanics, G. P. Galdi and J. Nečas (eds.), Res. Notes Math. Ser. 291, Longman, 129-162 (1993) Zbl 0818.76003 MR 1268237

[29] Rajagopal, K. R., Růžička, M.: Mathematical modeling of electrorheological materials. Continuum Mechanics and Thermodynamics 13, 59-78 (2001) Zbl 0971.76100

[30] Růžička, M.: Electrorheological Fluids: Modeling and Mathematical Theory. Lecture Notes in Math. 1748, Springer, Berlin (2000) Zbl 0962.76001 MR 1810360

[31] Serrin, J.: Mathematical principles of classical fluid mechanics. In: Encyclopedia of Physics VIII, Springer, Berlin, 125-263 (1959) MR 0108116

[32] Smagorinsky, J. S.: General circulation experiments with the primitive equations. I. The basic experiment. Mon. Weather Rev. 91, 99-164 (1963)

[33] Stokes, G.: Trans. Cambridge Philos. Soc. 8, 75-129 (1845)

[34] Zhikov, V. V.: Meyer-type estimates for solving the nonlinear Stokes system. Differential Equations 33, 108-115 (1997) Zbl 0911.35089 MR 1607245 